CRYSTALLOGRAPHIC COMMUNICATIONS

ISSN 2056-9890

Received 15 March 202

Accepted 9 April 2021

Edited by C. Schulzke, Universität Greifswald, Germany

Keywords: crystal structure; ketenylidene; trigold.

CCDC reference: 2070092

Supporting information: this article has supporting information at journals.iucr.org/e

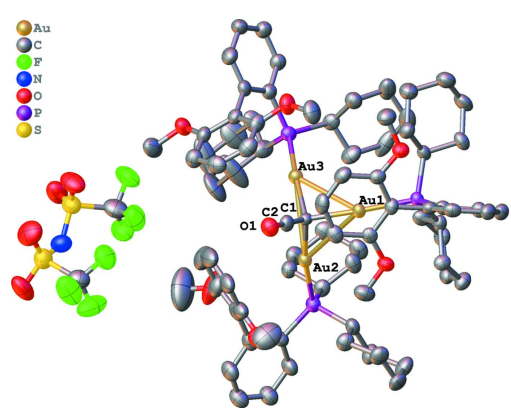

OPEN $\odot$ ACCESS

\section{Crystal structure of tris(2-dicyclohexylphosphino- $2^{\prime}, 6^{\prime}$-dimethoxy-1, 1'-biphenyl- $\kappa P$ )- $\mu$-oxoethenyl- idene-triangulo-trigold(I) bis(trifluoromethane- sulfonyl)imide}

\author{
Colin T. Hartgerink, ${ }^{a}$ Richard J. Staples ${ }^{\mathrm{b}}$ and Carolyn E. Anderson ${ }^{\mathrm{a} *}$
}

a Department of Chemistry and Biochemistry, Calvin University, 1726 Knollcrest Circle SE, Grand Rapids, MI 49546, USA,
and ${ }^{\mathbf{b} C e n t e r}$ for Crystallographic Research, Department of Chemistry, Michigan State University, 578 S. Shaw Lane, East
Lansing, MI 48824, USA. *Correspondence e-mail: cea3@calvin.edu

The title ketenylidene, $\left[\mathrm{Au}_{3}\left(\mathrm{C}_{2} \mathrm{O}\right)\left(\mathrm{C}_{26} \mathrm{H}_{35} \mathrm{O}_{2} \mathrm{P}\right)_{3}\right]\left(\mathrm{C}_{2} \mathrm{~F}_{6} \mathrm{NO}_{4} \mathrm{~S}_{2}\right)$, was obtained upon exposure of [2-(dicyclohexylphosphino)-2' ${ }^{\prime} 6^{\prime}$-dimethoxy-1,1'-biphenyl]gold(I) bis(trifluoromethanesulfonyl)imide to acetic anhydride at elevated temperature. The ketenylidene bridge caps the tri-gold cluster. The title compound has provided crystals that upon analysis represent the first tri-gold ketenylidene with atomic distances indicative of bonding interaction between the gold atoms.

\section{Chemical context}

Metal clusters containing ketenylidenes are of interest for their wide range of applications. For instance, ketenylidenes are useful for facilitating $\mathrm{C}-\mathrm{C}$ bond formation and cleavage (Went et al., 1987), metal cluster building (Sailor \& Shriver, 1985), and as potential intermediates for carbon monoxide chemistry (Jensen \& Shriver, 1992). One of the first transitionmetal ketenylidene complexes described was a tricobalt cluster reported by Seyferth et al. in 1974 (Seyferth et al., 1974). Since then, the scope of ketenylidene clusters has been expanded to include metals such as osmium (Went et al., 1987), ruthenium (Sailor \& Shriver, 1985), molybdenum (Ramalakshmi et al., 2015), and manganese (Crespi \& Shriver, 1986) to name a few.

However, relatively few ketenylidenes involving gold have been reported. Work by Green and co-workers uncovered a surface-bound gold ketenylidene $\left[\mathrm{Au}_{2} \mathrm{CCO}\right]$, which serves as a reactive intermediate in the aerobic oxidation of acetic acid on $\mathrm{Au} / \mathrm{TiO}_{2}$ surfaces (Green et al., 2012). More recently, Daugherty and co-workers reported the first instance of a tri-gold ketenylidene (Daugherty et al., 2017). In that case, the $\mathrm{Au} \cdots \mathrm{Au}$ distances suggest that there is no bonding interaction between the metal atoms.

Herein, we describe the first crystal structure analysis of a tri-gold ketenylidene in which the atomic distances suggest a bonding interaction between the gold atoms

\section{Structural commentary}

The molecular structure of the title compound is shown in Fig. 1. Four molecules are present in the unit cell $(Z=4)$ and there is one component in the asymmetric unit. The title 
compound consists of three molecules of (2-dicyclohexylphosphino-2',6'-dimethoxy-1,1'-biphenyl)gold(I) bis(trifluoromethanesulfonyl)imide capped by a ketenylidene unit $(\mathrm{C}=\mathrm{C}=\mathrm{O})$ to form a tri-gold cluster. The tri-gold cluster has an overall charge of +1 , with trifluoromethanesulfonylimide serving as the counter-ion. As shown in Fig. 1, the ketenylidene atoms $(\mathrm{C}=\mathrm{C}=\mathrm{O})$ form an angle of $88.1(5)^{\circ}$ with the mean $\mathrm{Au} 1-\mathrm{Au} 2-\mathrm{Au} 3$ gold plane.

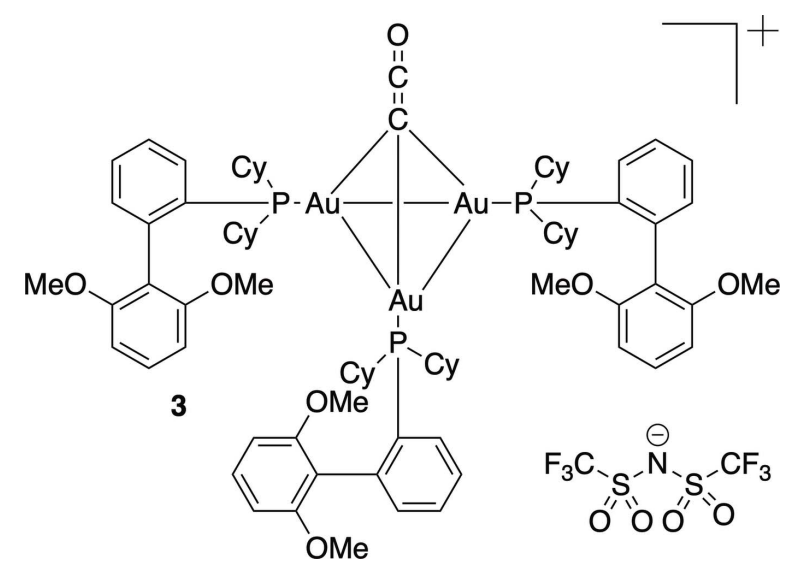

The $\mathrm{Au}-\mathrm{Au}$ bond distances suggest aurophilic interaction (Schmidbaur \& Schier, 2012). The shortest $\mathrm{Au}-\mathrm{Au}$ bond length is 3.1910 (5) $\AA$ (Au1-Au3), which is significantly shorter than the sum of two van der Waals radii (Bondi, 1964). The Au2-Au3 bond length of 3.2101 (5) $\AA$ also indicates a significant $\mathrm{Au}-\mathrm{Au}$ interaction, although the complex is not entirely symmetrical, with the $\mathrm{Au} 1-\mathrm{Au} 2$ bond length measuring 3.3005 (5) $\AA$. Other bond lengths within the cluster are more highly conserved within each subunit of the trimeric structure [e.g. $\mathrm{Au}-\mathrm{C} 1$ distances: 2.090 (7) to 2.098 (7) $\AA$; $\mathrm{Au}-\mathrm{P}$ distances: 2.273 (2) to 2.281 (2) $\mathrm{A}]$.

\section{Supramolecular features}

In the crystal structure of the title compound, the discrete complexes are arranged into columns along the $b$ axis (Fig. 2). Within these columns, the ketenylidene atoms alternate between the $+a$ and $-a$-axis directions. The only other similar cluster with $\mathrm{Au}$ was reported by Daugherty and co-workers (Daugherty et al., 2017), and it does not show this alternating arrangement. However, in this earlier case, the Au atoms are all bonded to the carbon of $N$-heterocyclic carbene ligands, rather than to phosphines. As such, the title compound is the first trimetallic ketenylidene cluster of any metal that involves the metal bound to only phosphine and the ketenylidiene bridge, rather than the more common $\mathrm{C}=\mathrm{O}$ ligand found in most trimetallic metal complexes in the CSD.

\section{Database survey}

The Cambridge Structural Database (CSD, Version 5.42, February 2021; Groom et al., 2016) contains 12 unique struc-

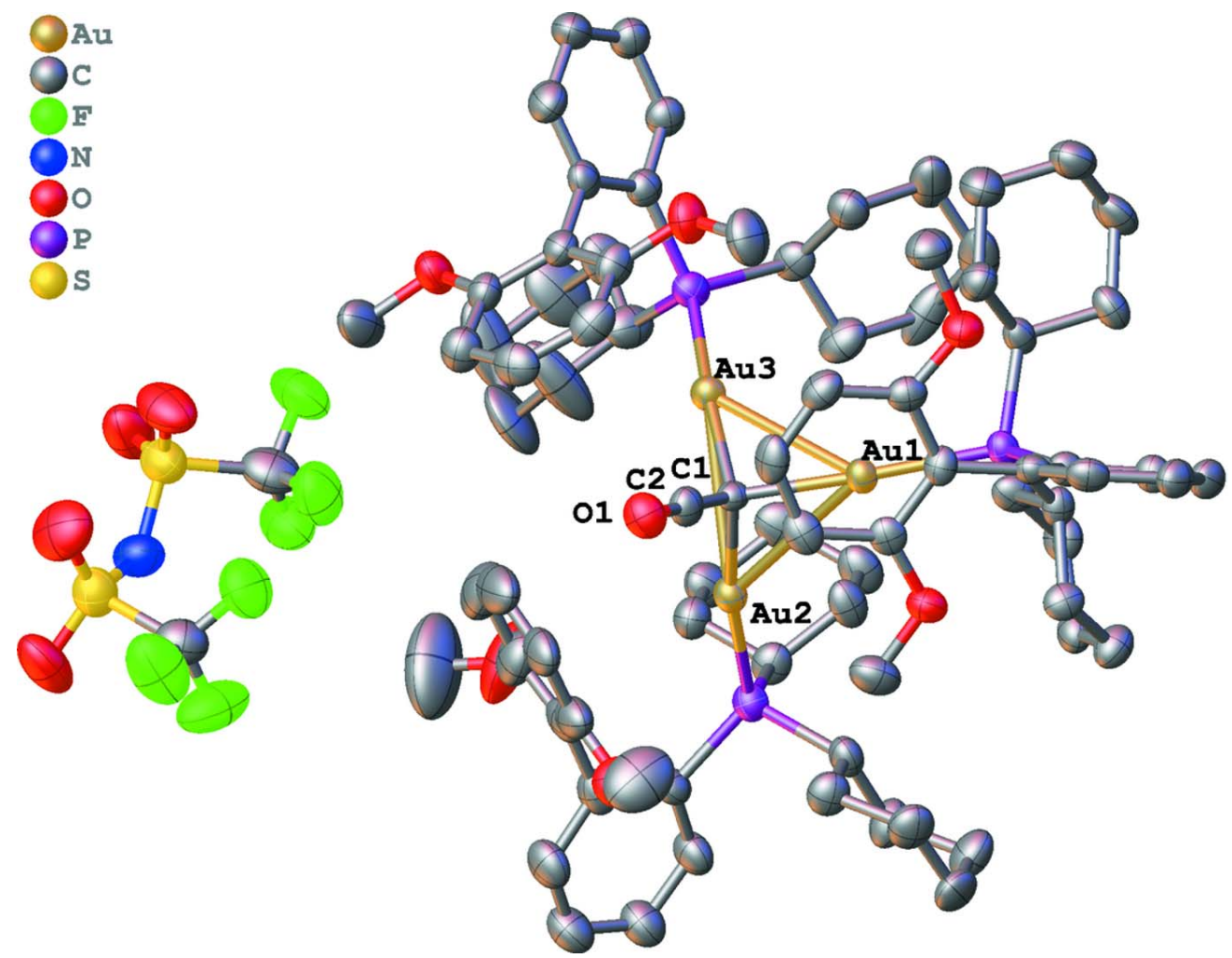

Figure 1

The molecular structure of the title compound with select atom labeling. Displacement ellipsoids are drawn at the $50 \%$ probability level. Hydrogen atoms are omitted for clarity reasons. 


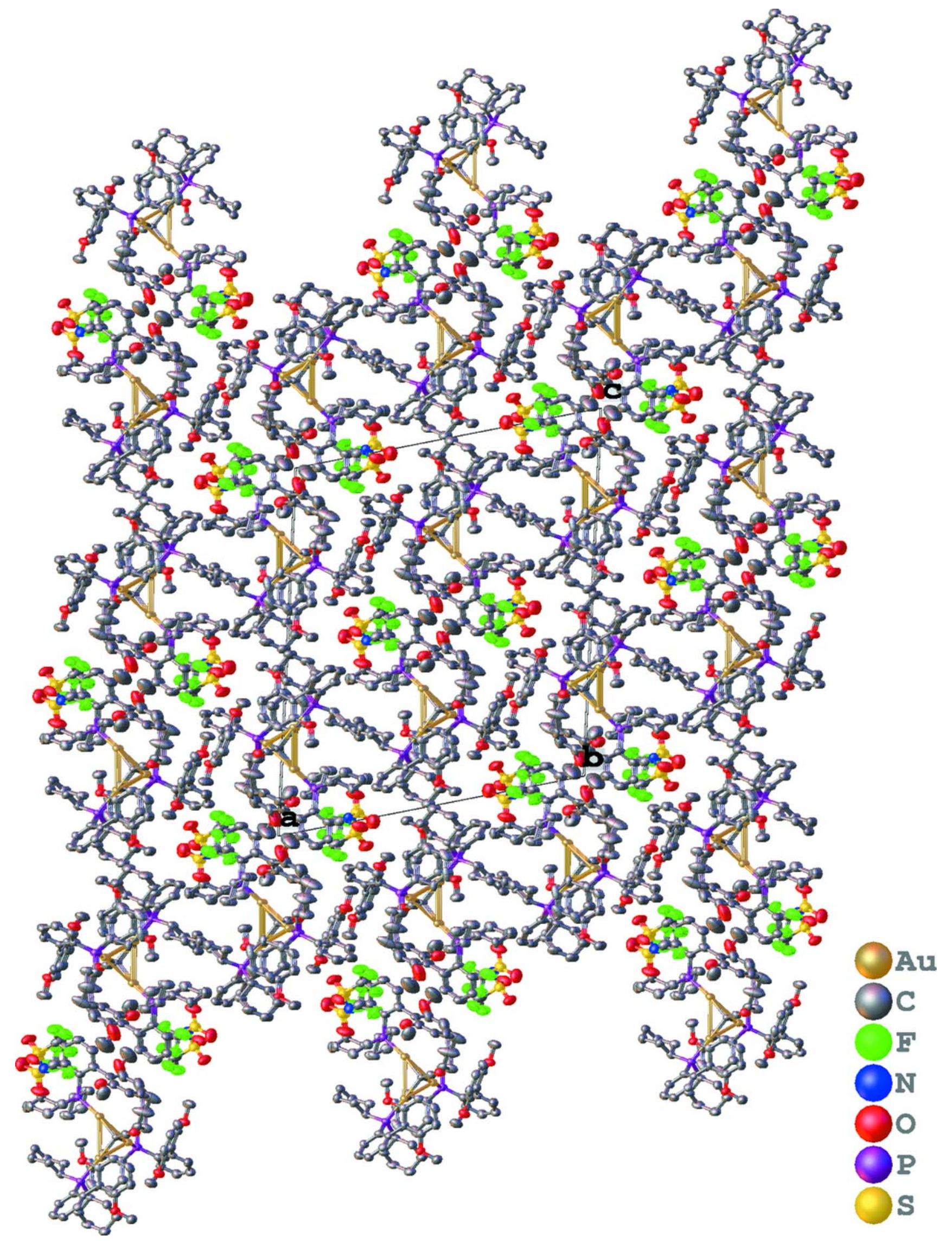


tures of ketenylidene-bridged metal clusters. Of these, ten contain clusters of three metal atoms, including: all iron (CAXVAY, Kolis et al., 1983; GEFPIQ, Bogdan et al., 1988), all ruthenium (DELSAO, Sailor \& Shriver, 1985; FONWAG, Sailor et al., 1987), all osmium (FONYEM, Went et al., 1987), and all gold(I) (LEBFOQ, Daugherty et al., 2017) and various combinations of iron, cobalt, manganese, molybdenum, and ruthenium (DUHDIT, Crespi \& Shriver, 1986 and Crespi et al., 1988; GAHBIA, Ching et al., 1988; HUQBIG, Ramalakshmi et al., 2015; KALVAU, Ching et al., 1989). Two additional structures of this type with central clusters of four metal atoms, either three ruthenium and one copper (PAJWOM, Gunale et al., 1992) or three iron and one copper (KINFOC10, Gunale et $a l ., 1992)$ have also been reported. Within this group, only one $2 \mathrm{Fe} / 1 \mathrm{Co}$ cluster bears a phosphine ligand (KALVAU, Ching et al., 1989) similar to the reported title compound; however, even in this case, the reported cluster is much simpler than the title compound, as all of the remaining positions are occupied by $\mathrm{CO}$.

The only other known all gold(I) cluster (LEBFOQ, Daugherty et al., 2017) differs from both the reported title compound as well as the other structures in the CSD, as it bears $N$-heterocyclic carbene ligands attached to gold, rather than either phosphines or carbon monoxide ligands. Additionally, the gold(I) atoms in this previously reported cluster were too far apart from each other to have any metal-metal bonding interaction.

Thus, the reported ketenylidene cluster differs from similar compounds in the CSD in both the title cluster's unique phosphine ligands and the short $\mathrm{Au}-\mathrm{Au}$ bonding interactions.

\section{Synthesis and crystallization}

The title compound was observed during scope studies related to the gold(I)-catalyzed synthesis of trisubstituted indolizine $\mathbf{2}$
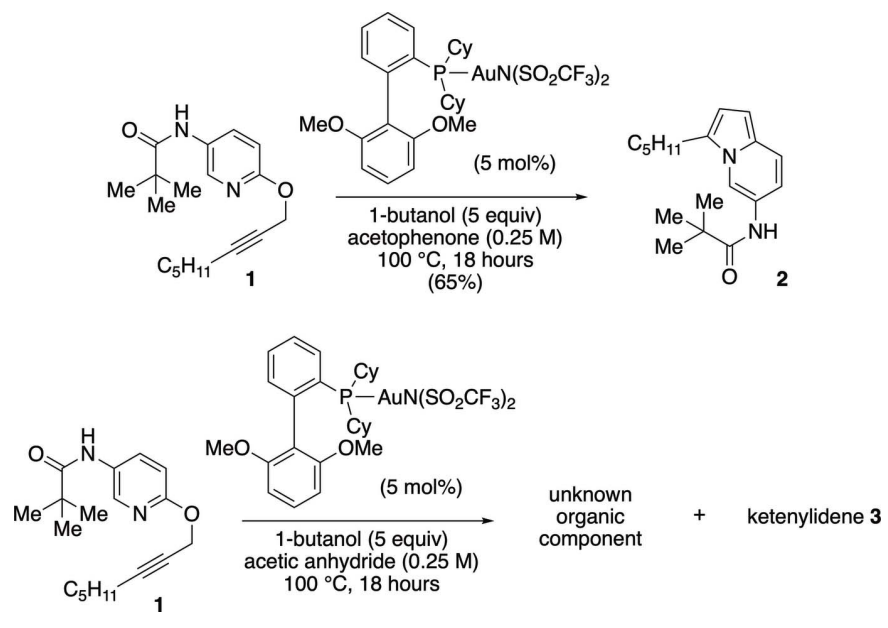

Figure 3

Reaction scheme. The title ketenylidene $\mathbf{3}$ was discovered as an unexpected by-product of a reaction exploring the gold(I)-catalyzed rearrangement of 2-propargyloxypyridine $\mathbf{1}$.
Table 1

Experimental details.

\begin{tabular}{|c|c|}
\hline \multicolumn{2}{|l|}{ Crystal data } \\
\hline Chemical formula & $\begin{array}{l}{\left[\mathrm{Au}_{3}\left(\mathrm{C}_{2} \mathrm{O}\right)\left(\mathrm{C}_{26} \mathrm{H}_{35} \mathrm{O}_{2} \mathrm{P}\right)_{3}\right]-} \\
\quad\left(\mathrm{C}_{2} \mathrm{~F}_{6} \mathrm{NO}_{4} \mathrm{~S}_{2}\right)\end{array}$ \\
\hline$M_{\mathrm{r}}$ & 2142.59 \\
\hline Crystal system, space group & Monoclinic, $P 2_{1} / n$ \\
\hline Temperature (K) & 173 \\
\hline$a, b, c(\AA)$ & $\begin{array}{l}24.0018(3), 12.4867(1) \\
\quad 28.4299(3)\end{array}$ \\
\hline$\beta\left(^{\circ}\right)$ & $103.7669(8)$ \\
\hline$V\left(\AA^{3}\right)$ & $8275.75(15)$ \\
\hline$Z$ & 4 \\
\hline Radiation type & $\mathrm{Cu} K \alpha$ \\
\hline$\mu\left(\mathrm{mm}^{-1}\right)$ & 11.42 \\
\hline Crystal size $(\mathrm{mm})$ & $0.2 \times 0.05 \times 0.03$ \\
\hline \multicolumn{2}{|l|}{ Data collection } \\
\hline Diffractometer & Bruker APEXII CCD \\
\hline Absorption correction & $\begin{array}{l}\text { Multi-scan (SADABS; Bruker, } \\
\text { 2013) }\end{array}$ \\
\hline$T_{\min }, T_{\max }$ & $0.321,0.735$ \\
\hline $\begin{array}{l}\text { No. of measured, independent and } \\
\text { observed }[I>2 \sigma(I)] \text { reflections }\end{array}$ & $73587,15716,11064$ \\
\hline$R_{\text {int }}$ & 0.110 \\
\hline$(\sin \theta / \lambda)_{\max }\left(\AA^{-1}\right)$ & 0.610 \\
\hline \multicolumn{2}{|l|}{ Refinement } \\
\hline$R\left[F^{2}>2 \sigma\left(F^{2}\right)\right], w R\left(F^{2}\right), S$ & $0.048,0.120,1.01$ \\
\hline No. of reflections & 15716 \\
\hline No. of parameters & 979 \\
\hline $\mathrm{H}$-atom treatment & $\mathrm{H}$-atom parameters constrained \\
\hline$\Delta \rho_{\max }, \Delta \rho_{\min }\left(\mathrm{e} \AA^{-3}\right)$ & $1.77,-0.95$ \\
\hline
\end{tabular}

Computer programs: COSMO and SAINT (Bruker, 2013), SHELXT (Sheldrick, 2015a), SHELXL2018/3 (Sheldrick, 2015b) and OLEX2 (Dolomanov et al., 2009).

from 2-propargyloxypyridine 1 (Rossler et al., 2019). While initial studies had shown that treatment of pyridine $\mathbf{1}$ with methyl ketones in the presence of alcohols and (2-dicyclohexylphosphino-2',6'-dimethoxy-1,1'-biphenyl)gold(I) bis(trifluoromethane-sulfonyl)imide could provide trisubstituted indolizines $\mathbf{2}$ in moderate to good yields, when the methyl ketone was replaced with acetic anhydride, an unknown organic product and the title ketenylidene cluster $\mathbf{3}$ were observed (Fig. 3). In an attempt to determine the organic product of the reaction, crystals were grown by the slow evaporation of a concentrated ethanol solution over several weeks at room temperature. Using this method, a few tiny yellow needle-shaped crystals, suitable for X-ray diffraction, were obtained and analyzed. However, rather than revealing the structure of the organic product as expected, the X-ray structure revealed the title ketenylidene-bridged tri-gold cluster 3. Subsequent studies aimed at the independent synthesis of cluster $\mathbf{3}$ and related species stoichiometrically were unsuccessful.

\section{Refinement}

Crystal data, data collection, and refinement details are collected in Table 1. All non-hydrogen atoms were refined anisotropically. Hydrogen-atom positions were calculated geometrically $(\mathrm{C}-\mathrm{H}=0.95-1.00 \AA)$ and refined using a riding model with $U_{\text {iso }}(\mathrm{H})=1.2 U_{\text {eq }}(\mathrm{C})$ or $1.5 U_{\text {eq }}(\mathrm{C}$-methyl $)$. 


\section{Acknowledgements}

The authors would like to thank Dr S. Biros for help with manuscript preparation. Funding for the Single Crystal X-ray diffractometer was provided by the MRI program by the National Science Foundation under grant No. 1919565.

\section{Funding information}

Funding for this research was provided by: National Science Foundation, Directorate for Mathematical and Physical Sciences (grant No. CHE-1665139 to C. Anderson and grant No. CHE-1919565 to R. Staples); Organic Syntheses, Inc. (grant No. Summer Research at an Undergraduate Institution to C. Hartgerink).

\section{References}

Bogdan, P. L., Sabat, M., Sunshine, S. A., Woodcock, C. \& Shriver, D. F. (1988). Inorg. Chem. 27, 1904-1910.

Bondi, A. (1964). J. Phys. Chem. 68, 441-451.

Bruker (2013). APEX2, SAINT and SADABS. Bruker AXS Inc. Madison, Wisconsin, USA.

Ching, S., Holt, E. M., Kolis, J. W. \& Shriver, D. F. (1988). Organometallics, 7, 892-898.

Ching, S., Sabat, M. \& Shriver, D. F. (1989). Organometallics, 8, $1047-$ 1058.

Crespi, A. M. \& Shriver, D. F. (1986). Organometallics, 5, 1750-1752.
Crespi, A. M., Went, M. J., Sunshine, S. S. \& Shriver, D. F. (1988). Organometallics, 7, 214-218.

Daugherty, N. T., Bacsa, J. \& Sadighi, J. P. (2017). Organometallics, 36, 3171-3174.

Dolomanov, O. V., Bourhis, L. J., Gildea, R. J., Howard, J. A. K. \& Puschmann, H. (2009). J. Appl. Cryst. 42, 339-341.

Green, I. X., Tang, W., Neurock, M. \& Yates, J. T. (2012). J. Am. Chem. Soc. 134, 13569-13572.

Groom, C. R., Bruno, I. J., Lightfoot, M. P. \& Ward, S. C. (2016). Acta Cryst. B72, 171-179.

Gunale, A. S., Jensen, M. P., Phillips, D. A., Stern, C. L. \& Shriver, D. F. (1992). Inorg. Chem. 31, 2622-2626.

Jensen, M. P. \& Shriver, D. F. (1992). J. Mol. Catal. 74, 73-84.

Kolis, J. W., Holt, E. M. \& Shriver, D. F. (1983). J. Am. Chem. Soc. 105, 7307-7313.

Ramalakshmi, R., Mondal, B., Bhattacharyya, M., Varghese, B. \& Ghosh, S. (2015). J. Organomet. Chem. 798, 106-111.

Rossler, M. D., Hartgerink, C. T., Zerull, E. E., Boss, B. L., Frndak, A. K., Mason, M. M., Nickerson, L. A., Romero, E. O., Van de Burg, J. E., Staples, R. J. \& Anderson, C. E. (2019). Org. Lett. 21, 5591-5595.

Sailor, M. J., Brock, C. P. \& Shriver, D. F. (1987). J. Am. Chem. Soc. 109, 6015-6022.

Sailor, M. J. \& Shriver, D. F. (1985). Organometallics, 4, 1476-1478.

Schmidbaur, H. \& Schier, A. (2012). Chem. Soc. Rev. 41, 370-412.

Seyferth, D., Hallgren, J. E. \& Eschbach, C. S. (1974). J. Am. Chem. Soc. 96, 1730-1737.

Sheldrick, G. M. (2015a). Acta Cryst. A71, 3-8.

Sheldrick, G. M. (2015b). Acta Cryst. C71, 3-8.

Went, M. J., Sailor, M. J., Bogdan, P. L., Brock, C. P. \& Shriver, D. F. (1987). J. Am. Chem. Soc. 109, 6023-6029. 


\section{supporting information}

Acta Cryst. (2021). E77, 537-541 [https://doi.org/10.1107/S2056989021003844]

Crystal structure of tris(2-dicyclohexylphosphino-2', 6'-dimethoxy-1,1'-biphenyl- $\kappa P$ )- $\mu$-oxoethenylidene-triangulo-trigold(I) bis(trifluoromethanesulfonyl)imide

\section{Colin T. Hartgerink, Richard J. Staples and Carolyn E. Anderson}

Computing details

Data collection: COSMO (Bruker, 2013); cell refinement: SAINT (Bruker, 2013); data reduction: SAINT (Bruker, 2013); program(s) used to solve structure: SHELXT (Sheldrick, 2015a); program(s) used to refine structure: SHELXL2018/3 (Sheldrick, 2015b); molecular graphics: Olex2 (Dolomanov et al., 2009); software used to prepare material for publication: Olex2 (Dolomanov et al., 2009).

Tris(2-dicyclohexylphosphino-2',6'-dimethoxy-1,1'-biphenyl- $\kappa P)$ - $\mu$-oxoethenylidene-triangulo-trigold(I) bis(trifluoromethanesulfonyl)imide

Crystal data

$\left[\mathrm{Au}_{3}\left(\mathrm{C}_{2} \mathrm{O}\right)\left(\mathrm{C}_{26} \mathrm{H}_{35} \mathrm{O}_{2} \mathrm{P}\right)_{3}\right]\left(\mathrm{C}_{2} \mathrm{~F}_{6} \mathrm{NO}_{4} \mathrm{~S}_{2}\right)$

$M_{r}=2142.59$

Monoclinic, $P 2{ }_{1} / n$

$a=24.0018(3) \AA$

$b=12.4867(1) \AA$

$c=28.4299(3) \AA$

$\beta=103.7669(8)^{\circ}$

$V=8275.75(15) \AA^{3}$

$Z=4$

\section{Data collection}

Bruker APEXII CCD diffractometer

Radiation source: sealed tube Graphite monochromator

Detector resolution: 8.36 pixels $\mathrm{mm}^{-1}$ $\omega$ and $\varphi$ scans

Absorption correction: multi-scan

(SADABS; Bruker, 2013)

$T_{\min }=0.321, T_{\max }=0.735$

Refinement

Refinement on $F^{2}$

Least-squares matrix: full

$R\left[F^{2}>2 \sigma\left(F^{2}\right)\right]=0.048$

$w R\left(F^{2}\right)=0.120$

$S=1.01$

15716 reflections
$F(000)=4240$

$D_{\mathrm{x}}=1.720 \mathrm{Mg} \mathrm{m}^{-3}$

$\mathrm{Cu} K \alpha$ radiation, $\lambda=1.54178 \AA$

Cell parameters from 9988 reflections

$\theta=2.2-70.1^{\circ}$

$\mu=11.42 \mathrm{~mm}^{-1}$

$T=173 \mathrm{~K}$

Needle, yellow

$0.2 \times 0.05 \times 0.03 \mathrm{~mm}$

73587 measured reflections

15716 independent reflections

11064 reflections with $I>2 \sigma(I)$

$R_{\text {int }}=0.110$

$\theta_{\text {max }}=70.1^{\circ}, \theta_{\text {min }}=2.2^{\circ}$

$h=-29 \rightarrow 28$

$k=-15 \rightarrow 15$

$l=-34 \rightarrow 34$

979 parameters

0 restraints

Primary atom site location: dual

Hydrogen site location: inferred from neighbouring sites

$\mathrm{H}$-atom parameters constrained 
$w=1 /\left[\sigma^{2}\left(F_{\mathrm{o}}{ }^{2}\right)+(0.0447 P)^{2}+20.5744 P\right]$

where $P=\left(F_{\mathrm{o}}^{2}+2 F_{\mathrm{c}}^{2}\right) / 3$

$(\Delta / \sigma)_{\max }=0.001$
$\Delta \rho_{\max }=1.77$ e $\AA^{-3}$

$\Delta \rho_{\min }=-0.95$ e $\AA^{-3}$

\section{Special details}

Experimental. Data was collected using a BRUKER CCD (charge coupled device) based diffractometer equipped with an Oxford low-temperature apparatus operating at $173 \mathrm{~K}$. A suitable crystal was chosen and mounted on a nylon loop using Paratone oil. Data were measured using omega scans of $0.5^{\circ}$ per frame for $30 \mathrm{~s}$. The total number of images were based on results from the program COSMO where redundancy was expected to be 4 and completeness to $0.83 \AA$ to $100 \%$. Cell parameters were retrieved using APEX II software and refined using SAINT on all observed reflections.Data reduction was performed using the SAINT software which corrects for Lp. Scaling and absorption corrections were applied using SADABS6 multi-scan technique, supplied by George Sheldrick. The structure was solved by the direct method using the SHELXT program and refined by least squares method on F2, SHELXL, incorporated in OLEX2.

Geometry. All esds (except the esd in the dihedral angle between two 1.s. planes) are estimated using the full covariance matrix. The cell esds are taken into account individually in the estimation of esds in distances, angles and torsion angles; correlations between esds in cell parameters are only used when they are defined by crystal symmetry. An approximate (isotropic) treatment of cell esds is used for estimating esds involving 1.s. planes.

Refinement. The structure was refined by Least Squares using version 2014/6 of XL (Sheldrick, 2008) incorporated in Olex2 (Dolomanov et al., 2009). All non-hydrogen atoms were refined anisotropically. Hydrogen atom positions were calculated geometrically and refined using the riding model.'

Fractional atomic coordinates and isotropic or equivalent isotropic displacement parameters $\left(\hat{A}^{2}\right)$

\begin{tabular}{|c|c|c|c|c|}
\hline & $x$ & $y$ & $z$ & $U_{\text {iso }} * / U_{\text {eq }}$ \\
\hline $\mathrm{Au} 1$ & $0.53937(2)$ & 0.36171 & $0.21151(2)$ & $0.03027(9)$ \\
\hline $\mathrm{Au} 2$ & $0.54502(2)$ & $0.30927(3)$ & $0.32634(2)$ & $0.03393(9)$ \\
\hline Au3 & $0.44934(2)$ & $0.20237(3)$ & $0.24086(2)$ & $0.03374(9)$ \\
\hline $\mathrm{P} 1$ & $0.59759(9)$ & $0.34972(16)$ & $0.15898(8)$ & $0.0314(4)$ \\
\hline $\mathrm{P} 2$ & $0.61858(10)$ & $0.26651(18)$ & $0.39059(8)$ & $0.0368(5)$ \\
\hline P3 & $0.41808(10)$ & $0.03219(17)$ & $0.22248(8)$ & $0.0364(5)$ \\
\hline O1 & $0.4258(3)$ & $0.5067(5)$ & $0.2643(2)$ & $0.0507(16)$ \\
\hline $\mathrm{O} 2$ & 0.5926 & $0.6146(5)$ & $0.2136(2)$ & $0.0412(14)$ \\
\hline $\mathrm{O} 3$ & $0.4780(3)$ & $0.5587(5)$ & $0.0590(2)$ & $0.0425(14)$ \\
\hline $\mathrm{O} 4$ & $0.4879(4)$ & $0.2011(9)$ & $0.4500(5)$ & $0.118(4)$ \\
\hline $\mathrm{O} 5$ & $0.5453(4)$ & $0.5423(6)$ & $0.4146(3)$ & $0.071(2)$ \\
\hline O6 & $0.2659(3)$ & $0.1195(5)$ & $0.2662(2)$ & $0.0518(17)$ \\
\hline $\mathrm{O} 7$ & $0.3411(3)$ & $0.2431(6)$ & 0.1389 (3) & $0.0555(18)$ \\
\hline $\mathrm{C} 1$ & 0.4875 & $0.3511(6)$ & $0.2611(3)$ & $0.0274(16)$ \\
\hline $\mathrm{C} 2$ & $0.4552(4)$ & $0.4319(7)$ & $0.2631(3)$ & $0.038(2)$ \\
\hline $\mathrm{C} 3$ & $0.6180(4)$ & $0.4679(6)$ & $0.1282(3)$ & $0.0336(18)$ \\
\hline $\mathrm{C} 4$ & $0.6653(4)$ & $0.4587(7)$ & $0.1086(3)$ & 0.0367 (19) \\
\hline $\mathrm{H} 4$ & 0.688034 & 0.395750 & 0.115090 & $0.044^{*}$ \\
\hline $\mathrm{C} 5$ & $0.6808(4)$ & $0.5367(7)$ & $0.0801(3)$ & $0.043(2)$ \\
\hline H5 & 0.713897 & 0.527777 & 0.067595 & $0.052 *$ \\
\hline C6 & $0.6482(4)$ & $0.6270(8)$ & 0.0698 & $0.043(2)$ \\
\hline H6 & 0.657838 & 0.680655 & 0.049466 & $0.052 *$ \\
\hline $\mathrm{C} 7$ & $0.6006(4)$ & $0.6402(7)$ & 0.0894 & $0.040(2)$ \\
\hline H7 & 0.578703 & 0.704180 & 0.082893 & $0.048 *$ \\
\hline $\mathrm{C} 8$ & 0.5845 & $0.5616(6)$ & $0.1184(3)$ & $0.0330(18)$ \\
\hline C9 & $0.5331(3)$ & $0.5833(6)$ & $0.1374(3)$ & $0.0340(18)$ \\
\hline
\end{tabular}




\begin{tabular}{|c|c|c|c|c|}
\hline $\mathrm{C} 10$ & $0.5377(4)$ & $0.6131(6)$ & $0.1857(3)$ & $0.0339(18)$ \\
\hline $\mathrm{C} 11$ & $0.4892(4)$ & $0.6375(7)$ & $0.2022(3)$ & $0.043(2)$ \\
\hline H11 & 0.492373 & 0.655634 & 0.235195 & $0.052 *$ \\
\hline $\mathrm{C} 12$ & $0.4365(4)$ & $0.6351(7)$ & $0.1700(4)$ & $0.046(2)$ \\
\hline H12 & 0.403331 & 0.652251 & 0.181156 & $0.055^{*}$ \\
\hline $\mathrm{C} 13$ & $0.4304(4)$ & $0.6090(7)$ & $0.1224(4)$ & $0.044(2)$ \\
\hline H13 & 0.393527 & 0.607929 & 0.100791 & $0.053 *$ \\
\hline $\mathrm{C} 14$ & $0.4790(4)$ & $0.5838(6)$ & $0.1060(3)$ & 0.0366 (19) \\
\hline $\mathrm{C} 15$ & $0.6001(5)$ & $0.6310(8)$ & $0.2644(4)$ & $0.058(3)$ \\
\hline H15A & 0.584512 & 0.701133 & 0.270078 & $0.087^{*}$ \\
\hline H15B & 0.641006 & 0.628363 & 0.280373 & $0.087^{*}$ \\
\hline $\mathrm{H} 15 \mathrm{C}$ & 0.579768 & 0.574788 & 0.277735 & $0.087^{*}$ \\
\hline $\mathrm{C} 16$ & $0.4226(4)$ & $0.5417(9)$ & $0.0267(4)$ & $0.056(3)$ \\
\hline H16A & 0.427567 & 0.514674 & -0.004380 & $0.083^{*}$ \\
\hline H16B & 0.401483 & 0.609579 & 0.021584 & $0.083 *$ \\
\hline $\mathrm{H} 16 \mathrm{C}$ & 0.400999 & 0.489350 & 0.040980 & $0.083^{*}$ \\
\hline $\mathrm{C} 17$ & $0.5643(4)$ & $0.2573(7)$ & $0.1096(3)$ & $0.0347(18)$ \\
\hline H17 & 0.554779 & 0.190324 & 0.125365 & $0.042^{*}$ \\
\hline $\mathrm{C} 18$ & 0.6013 (4) & $0.2237(7)$ & $0.0751(3)$ & $0.044(2)$ \\
\hline H18A & 0.611749 & 0.287751 & 0.058530 & $0.053^{*}$ \\
\hline H18B & 0.637162 & 0.190220 & 0.093769 & $0.053^{*}$ \\
\hline C19 & $0.5692(5)$ & $0.1446(8)$ & $0.0374(4)$ & $0.057(3)$ \\
\hline H19A & 0.593181 & 0.126978 & 0.014542 & $0.068^{*}$ \\
\hline H19B & 0.562425 & 0.077565 & 0.053808 & $0.068^{*}$ \\
\hline $\mathrm{C} 20$ & $0.5121(5)$ & $0.1897(9)$ & 0.0094 (4) & $0.057(3)$ \\
\hline $\mathrm{H} 20 \mathrm{~A}$ & 0.519205 & 0.251229 & -0.010338 & $0.068^{*}$ \\
\hline H20B & 0.491462 & 0.134176 & -0.013001 & $0.068^{*}$ \\
\hline $\mathrm{C} 21$ & $0.4745(5)$ & $0.2260(9)$ & $0.0425(4)$ & $0.057(3)$ \\
\hline $\mathrm{H} 21 \mathrm{~A}$ & 0.462137 & 0.162899 & 0.058536 & $0.069^{*}$ \\
\hline H21B & 0.439727 & 0.261723 & 0.023096 & $0.069^{*}$ \\
\hline $\mathrm{C} 22$ & $0.5074(4)$ & $0.3038(7)$ & $0.0810(4)$ & $0.044(2)$ \\
\hline $\mathrm{H} 22 \mathrm{~A}$ & 0.514978 & 0.370838 & 0.065036 & $0.053^{*}$ \\
\hline H22B & 0.483260 & 0.321855 & 0.103709 & $0.053^{*}$ \\
\hline $\mathrm{C} 23$ & $0.6663(4)$ & $0.2883(6)$ & $0.1892(3)$ & $0.0347(18)$ \\
\hline $\mathrm{H} 23$ & 0.687237 & 0.268777 & 0.163914 & $0.042 *$ \\
\hline $\mathrm{C} 24$ & $0.6562(4)$ & $0.1845(6)$ & $0.2160(3)$ & $0.041(2)$ \\
\hline $\mathrm{H} 24 \mathrm{~A}$ & 0.636134 & 0.202298 & 0.241689 & $0.049^{*}$ \\
\hline H24B & 0.631552 & 0.134861 & 0.192923 & $0.049 *$ \\
\hline $\mathrm{C} 25$ & $0.7139(4)$ & $0.1300(7)$ & $0.2387(4)$ & $0.046(2)$ \\
\hline $\mathrm{H} 25 \mathrm{~A}$ & 0.706969 & 0.065621 & 0.256844 & $0.056^{*}$ \\
\hline H25B & 0.732180 & 0.106649 & 0.212692 & $0.056^{*}$ \\
\hline $\mathrm{C} 26$ & $0.7544(4)$ & $0.2061(8)$ & $0.2731(3)$ & $0.043(2)$ \\
\hline $\mathrm{H} 26 \mathrm{~A}$ & 0.738786 & 0.220874 & 0.301645 & $0.052 *$ \\
\hline H26B & 0.792276 & 0.171329 & 0.284525 & $0.052 *$ \\
\hline $\mathrm{C} 27$ & $0.7619(4)$ & $0.3117(8)$ & $0.2480(4)$ & $0.048(2)$ \\
\hline $\mathrm{H} 27 \mathrm{~A}$ & 0.785333 & 0.361275 & 0.271920 & $0.058^{*}$ \\
\hline H27B & 0.782679 & 0.298093 & 0.222420 & $0.058^{*}$ \\
\hline $\mathrm{C} 28$ & $0.7043(4)$ & $0.3639(7)$ & $0.2255(3)$ & $0.0383(19)$ \\
\hline
\end{tabular}




\begin{tabular}{|c|c|c|c|c|}
\hline $\mathrm{H} 28 \mathrm{~A}$ & 0.710730 & 0.430873 & 0.208902 & $0.046^{*}$ \\
\hline H28B & 0.684717 & 0.382670 & 0.251365 & $0.046^{*}$ \\
\hline $\mathrm{C} 29$ & $0.6169(4)$ & $0.3013(7)$ & $0.4529(3)$ & $0.040(2)$ \\
\hline $\mathrm{C} 30$ & $0.6664(4)$ & $0.2764(8)$ & $0.4899(4)$ & $0.051(2)$ \\
\hline $\mathrm{H} 30$ & 0.696362 & 0.235542 & 0.482178 & $0.061^{*}$ \\
\hline $\mathrm{C} 31$ & $0.6715(5)$ & $0.3106(9)$ & $0.5367(4)$ & $0.058(3)$ \\
\hline H31 & 0.704642 & 0.293109 & 0.561268 & $0.069 *$ \\
\hline $\mathrm{C} 32$ & $0.6277(5)$ & $0.3712(9)$ & $0.5478(4)$ & $0.060(3)$ \\
\hline $\mathrm{H} 32$ & 0.631325 & 0.397685 & 0.579737 & $0.072^{*}$ \\
\hline $\mathrm{C} 33$ & $0.5792(5)$ & $0.3923(8)$ & $0.5125(4)$ & $0.058(3)$ \\
\hline H33 & 0.549451 & 0.433181 & 0.520603 & $0.069 *$ \\
\hline $\mathrm{C} 34$ & $0.5723(4)$ & $0.3561(8)$ & $0.4655(4)$ & $0.049(2)$ \\
\hline $\mathrm{C} 35$ & 0.5158 & $0.3740(9)$ & $0.4305(4)$ & $0.052(3)$ \\
\hline $\mathrm{C} 36$ & $0.4742(5)$ & $0.2971(11)$ & $0.4246(5)$ & $0.071(3)$ \\
\hline $\mathrm{C} 37$ & $0.4210(6)$ & $0.3079(13)$ & $0.3935(6)$ & $0.090(5)$ \\
\hline H37 & 0.393316 & 0.252199 & 0.389193 & $0.108^{*}$ \\
\hline C38 & $0.4098(5)$ & $0.4049(13)$ & $0.3686(5)$ & $0.082(4)$ \\
\hline $\mathrm{H} 38$ & 0.373598 & 0.414986 & 0.346543 & $0.098 *$ \\
\hline C39 & $0.4489(5)$ & $0.4852(10)$ & $0.3748(4)$ & $0.061(3)$ \\
\hline H39 & 0.440122 & 0.551058 & 0.357920 & $0.073^{*}$ \\
\hline $\mathrm{C} 40$ & $0.5020(4)$ & $0.4694(10)$ & $0.4064(4)$ & $0.058(3)$ \\
\hline C41 & $0.4611(9)$ & $0.1637(19)$ & $0.4802(8)$ & 0.178 \\
\hline H41A & 0.454369 & 0.086915 & 0.474341 & $0.268 *$ \\
\hline H41B & 0.424264 & 0.200710 & 0.476118 & $0.268^{*}$ \\
\hline $\mathrm{H} 41 \mathrm{C}$ & 0.484121 & 0.174786 & 0.513242 & $0.268 *$ \\
\hline C42 & $0.5349(7)$ & $0.6405(12)$ & $0.3875(6)$ & $0.106(5)$ \\
\hline $\mathrm{H} 42 \mathrm{~A}$ & 0.531818 & 0.625531 & 0.353136 & $0.160^{*}$ \\
\hline H42B & 0.566778 & 0.690217 & 0.399398 & $0.160^{*}$ \\
\hline $\mathrm{H} 42 \mathrm{C}$ & 0.499126 & 0.672768 & 0.391507 & $0.160 *$ \\
\hline C43 & $0.6354(4)$ & $0.1216(7)$ & $0.3930(4)$ & $0.043(2)$ \\
\hline $\mathrm{H} 43$ & 0.669434 & 0.108234 & 0.420426 & $0.052 *$ \\
\hline $\mathrm{C} 44$ & $0.5838(4)$ & $0.0590(7)$ & $0.4024(4)$ & $0.045(2)$ \\
\hline $\mathrm{H} 44 \mathrm{~A}$ & 0.575897 & 0.082988 & 0.433312 & $0.054^{*}$ \\
\hline H44B & 0.549456 & 0.074185 & 0.376123 & $0.054^{*}$ \\
\hline $\mathrm{C} 45$ & $0.5956(5)$ & $-0.0607(8)$ & $0.4046(4)$ & $0.053(3)$ \\
\hline $\mathrm{H} 45 \mathrm{~A}$ & 0.628158 & -0.076379 & 0.432397 & $0.064^{*}$ \\
\hline H45B & 0.561465 & -0.099116 & 0.409858 & $0.064 *$ \\
\hline C46 & $0.6095(5)$ & $-0.1007(7)$ & $0.3584(4)$ & 0.059 (3) \\
\hline $\mathrm{H} 46 \mathrm{~A}$ & 0.575521 & -0.092007 & 0.331002 & $0.070^{*}$ \\
\hline H46B & 0.619145 & -0.177892 & 0.361620 & $0.070^{*}$ \\
\hline $\mathrm{C} 47$ & $0.6599(5)$ & $-0.0383(7)$ & $0.3481(4)$ & $0.055(3)$ \\
\hline H47A & 0.667039 & -0.062736 & 0.316940 & $0.066^{*}$ \\
\hline H47B & 0.694749 & -0.053823 & 0.373861 & $0.066^{*}$ \\
\hline $\mathrm{C} 48$ & $0.6493(5)$ & $0.0829(7)$ & $0.3458(4)$ & $0.048(2)$ \\
\hline H48A & 0.616908 & 0.099917 & 0.318028 & $0.057^{*}$ \\
\hline H48B & 0.683828 & 0.120460 & 0.340921 & $0.057^{*}$ \\
\hline C49 & $0.6807(4)$ & $0.3412(7)$ & $0.3790(3)$ & $0.042(2)$ \\
\hline $\mathrm{H} 49$ & 0.681878 & 0.324040 & 0.344894 & $0.051 *$ \\
\hline
\end{tabular}




\begin{tabular}{|c|c|c|c|c|}
\hline $\mathrm{C} 50$ & 0.7410 & $0.3146(8)$ & $0.4104(4)$ & $0.052(2)$ \\
\hline H50A & 0.743247 & 0.334052 & 0.444534 & $0.062 *$ \\
\hline H50B & 0.748318 & 0.236771 & 0.408944 & $0.062 *$ \\
\hline C51 & $0.7866(4)$ & $0.3773(9)$ & $0.3916(5)$ & $0.061(3)$ \\
\hline H51A & 0.825119 & 0.361399 & 0.412326 & $0.073^{*}$ \\
\hline H51B & 0.786035 & 0.353851 & 0.358255 & $0.073^{*}$ \\
\hline C52 & $0.7760(4)$ & $0.4959(8)$ & $0.3918(4)$ & $0.055(3)$ \\
\hline H52A & 0.804607 & 0.533685 & 0.377895 & $0.066^{*}$ \\
\hline H52B & 0.780626 & 0.520844 & 0.425589 & $0.066^{*}$ \\
\hline $\mathrm{C} 53$ & 0.7158 & $0.5233(8)$ & $0.3625(4)$ & $0.056(3)$ \\
\hline H53A & 0.712916 & 0.506597 & 0.327995 & $0.067^{*}$ \\
\hline H53B & 0.709109 & 0.601050 & 0.365162 & $0.067^{*}$ \\
\hline $\mathrm{C} 54$ & $0.6692(4)$ & $0.4610(7)$ & $0.3799(4)$ & 0.049 (2) \\
\hline H54A & 0.668976 & 0.483412 & 0.413288 & $0.059 *$ \\
\hline H54B & 0.631127 & 0.477376 & 0.358665 & $0.059^{*}$ \\
\hline C55 & $0.3468(4)$ & $0.0062(7)$ & 0.1833 & $0.0379(19)$ \\
\hline C56 & $0.3376(4)$ & $-0.0890(7)$ & $0.1569(3)$ & $0.043(2)$ \\
\hline H56 & 0.368843 & -0.136941 & 0.158874 & $0.051^{*}$ \\
\hline C57 & $0.2845(4)$ & $-0.1157(8)$ & $0.1280(4)$ & $0.048(2)$ \\
\hline H57 & 0.279522 & -0.180323 & 0.109843 & $0.058^{*}$ \\
\hline C58 & $0.2396(4)$ & $-0.0482(8)$ & $0.1260(4)$ & $0.052(3)$ \\
\hline $\mathrm{H} 58$ & 0.202989 & -0.065319 & 0.106163 & $0.062 *$ \\
\hline C59 & $0.2471(4)$ & $0.0462(9)$ & $0.1531(4)$ & $0.051(2)$ \\
\hline H59 & 0.214942 & 0.090974 & 0.152273 & $0.061^{*}$ \\
\hline C60 & $0.2999(4)$ & $0.0761(7)$ & $0.1808(3)$ & $0.038(2)$ \\
\hline C61 & $0.3043(4)$ & $0.1850(7)$ & $0.2036(3)$ & $0.040(2)$ \\
\hline C62 & 0.2853 & $0.2068(7)$ & $0.2452(3)$ & $0.041(2)$ \\
\hline C63 & $0.2845(4)$ & $0.3103(8)$ & $0.2625(4)$ & $0.052(2)$ \\
\hline H63 & 0.270172 & 0.324800 & 0.290286 & $0.063^{*}$ \\
\hline C64 & $0.3049(4)$ & $0.3914(8)$ & $0.2387(4)$ & $0.050(2)$ \\
\hline H64 & 0.305709 & 0.462204 & 0.251004 & $0.060 *$ \\
\hline C65 & $0.3242(4)$ & $0.3736(7)$ & $0.1976(4)$ & $0.051(2)$ \\
\hline H65 & 0.337491 & 0.431394 & 0.181520 & $0.061^{*}$ \\
\hline C66 & $0.3239(4)$ & $0.2710(7)$ & $0.1802(4)$ & $0.044(2)$ \\
\hline C67 & $0.2525(6)$ & $0.1374(10)$ & $0.3121(4)$ & 0.070 \\
\hline H67A & 0.218638 & 0.183715 & 0.307596 & $0.105^{*}$ \\
\hline H67B & 0.285073 & 0.172065 & 0.334161 & $0.105^{*}$ \\
\hline $\mathrm{H} 67 \mathrm{C}$ & 0.244473 & 0.068721 & 0.325783 & $0.105^{*}$ \\
\hline C68 & $0.3657(5)$ & $0.3256(9)$ & 0.1159 (4) & $0.068(3)$ \\
\hline H68A & 0.376332 & 0.296503 & 0.087230 & $0.102^{*}$ \\
\hline H68B & 0.399909 & 0.353722 & 0.138478 & $0.102^{*}$ \\
\hline H68C & 0.337692 & 0.383396 & 0.106079 & $0.102 *$ \\
\hline C69 & 0.4678 (4) & $-0.0396(7)$ & $0.1926(3)$ & $0.041(2)$ \\
\hline H69 & 0.458485 & -0.117645 & 0.192114 & $0.049 *$ \\
\hline $\mathrm{C} 70$ & $0.5302(4)$ & $-0.0252(8)$ & $0.2206(4)$ & $0.056(3)$ \\
\hline $\mathrm{H} 70 \mathrm{~A}$ & 0.534730 & -0.049412 & 0.254429 & $0.067^{*}$ \\
\hline H70B & 0.540854 & 0.051437 & 0.221069 & $0.067^{*}$ \\
\hline C71 & $0.5697(4)$ & $-0.0910(8)$ & $0.1962(5)$ & $0.071(4)$ \\
\hline
\end{tabular}




\begin{tabular}{|c|c|c|c|c|}
\hline $\mathrm{H} 71 \mathrm{~A}$ & 0.610169 & -0.077509 & 0.213111 & $0.085^{*}$ \\
\hline H71B & 0.561906 & -0.168218 & 0.199365 & $0.085^{*}$ \\
\hline $\mathrm{C} 72$ & $0.5613(5)$ & $-0.0634(8)$ & $0.1429(5)$ & $0.071(4)$ \\
\hline $\mathrm{H} 72 \mathrm{~A}$ & 0.576272 & 0.009536 & 0.140059 & $0.085^{*}$ \\
\hline $\mathrm{H} 72 \mathrm{~B}$ & 0.583877 & -0.113960 & 0.128011 & $0.085^{*}$ \\
\hline $\mathrm{C} 73$ & $0.4995(5)$ & $-0.0681(9)$ & $0.1153(5)$ & $0.069(4)$ \\
\hline $\mathrm{H} 73 \mathrm{~A}$ & 0.486483 & -0.143518 & 0.112594 & $0.083^{*}$ \\
\hline H73B & 0.496094 & -0.040098 & 0.082154 & $0.083 *$ \\
\hline $\mathrm{C} 74$ & $0.4611(4)$ & $-0.0027(7)$ & $0.1402(3)$ & $0.048(2)$ \\
\hline $\mathrm{H} 74 \mathrm{~A}$ & 0.420620 & -0.010943 & 0.122242 & $0.057^{*}$ \\
\hline H74B & 0.471363 & 0.074001 & 0.139774 & $0.057^{*}$ \\
\hline $\mathrm{C} 75$ & $0.4208(5)$ & $-0.0479(9)$ & $0.2776(4)$ & $0.057(3)$ \\
\hline H75 & 0.462643 & -0.061847 & 0.290921 & $0.069^{*}$ \\
\hline $\mathrm{C} 76$ & $0.3946(6)$ & $-0.1572(8)$ & $0.2695(4)$ & $0.061(3)$ \\
\hline H76A & 0.413062 & -0.196718 & 0.247087 & $0.073 *$ \\
\hline $\mathrm{H} 76 \mathrm{~B}$ & 0.353463 & -0.149391 & 0.253436 & $0.073^{*}$ \\
\hline $\mathrm{C} 77$ & $0.3998(8)$ & $-0.2226(10)$ & $0.3145(5)$ & $0.100(5)$ \\
\hline $\mathrm{H} 77 \mathrm{~A}$ & 0.375486 & -0.287293 & 0.306663 & $0.120^{*}$ \\
\hline H77B & 0.440066 & -0.246404 & 0.326290 & $0.120^{*}$ \\
\hline C78 & $0.3830(9)$ & $-0.1643(11)$ & $0.3516(6)$ & $0.131(8)$ \\
\hline $\mathrm{H} 78 \mathrm{~A}$ & 0.391791 & -0.208200 & 0.381419 & $0.157^{*}$ \\
\hline H78B & 0.340910 & -0.154361 & 0.342031 & $0.157^{*}$ \\
\hline C79 & $0.4115(8)$ & $-0.0526(13)$ & $0.3637(5)$ & $0.105(6)$ \\
\hline $\mathrm{H} 79 \mathrm{~A}$ & 0.393233 & -0.013570 & 0.386334 & $0.126^{*}$ \\
\hline H79B & 0.452864 & -0.060859 & 0.379026 & $0.126^{*}$ \\
\hline $\mathrm{C} 80$ & $0.4034(10)$ & $0.0097(10)$ & $0.3158(5)$ & $0.127(8)$ \\
\hline $\mathrm{H} 80 \mathrm{~A}$ & 0.362459 & 0.029214 & 0.304421 & $0.152 *$ \\
\hline H80B & 0.425722 & 0.077068 & 0.322131 & $0.152 *$ \\
\hline S1A & $0.24408(13)$ & $0.2219(2)$ & $0.52100(11)$ & $0.0607(7)$ \\
\hline $\mathrm{S} 2 \mathrm{~A}$ & $0.24474(12)$ & $0.0341(3)$ & $0.46694(11)$ & $0.0639(7)$ \\
\hline F1A & $0.3480(4)$ & $0.2976(7)$ & $0.5371(3)$ & $0.112(3)$ \\
\hline $\mathrm{F} 2 \mathrm{~A}$ & $0.2805(5)$ & $0.4110(6)$ & $0.5073(3)$ & $0.110(3)$ \\
\hline F3A & $0.3042(3)$ & $0.2920(6)$ & $0.4621(3)$ & $0.086(2)$ \\
\hline F4A & $0.3499(3)$ & $-0.0314(6)$ & $0.4807(3)$ & $0.081(2)$ \\
\hline F5A & $0.2957(4)$ & $-0.0660(7)$ & $0.4113(3)$ & $0.092(2)$ \\
\hline F6A & $0.3264(4)$ & $0.0971(6)$ & $0.4288(3)$ & 0.095 \\
\hline O1A & 0.1904 (4) & $0.2535(8)$ & $0.4885(4)$ & $0.096(3)$ \\
\hline $\mathrm{O} 2 \mathrm{~A}$ & $0.2535(5)$ & $0.2361(7)$ & $0.5724(3)$ & $0.092(3)$ \\
\hline $\mathrm{O} 3 \mathrm{~A}$ & $0.2090(4)$ & $0.0861(8)$ & 0.4249 (3) & $0.086(3)$ \\
\hline $\mathrm{O} 4 \mathrm{~A}$ & $0.2313(4)$ & $-0.0722(7)$ & $0.4819(3)$ & 0.085 \\
\hline N1A & $0.2676(4)$ & $0.1084(7)$ & $0.5117(4)$ & $0.065(3)$ \\
\hline $\mathrm{C} 1 \mathrm{~A}$ & $0.2971(6)$ & $0.3084(10)$ & $0.5070(5)$ & 0.068 \\
\hline $\mathrm{C} 2 \mathrm{~A}$ & $0.3075(6)$ & $0.0072(11)$ & $0.4477(4)$ & $0.073(4)$ \\
\hline
\end{tabular}

Atomic displacement parameters $\left(\AA^{2}\right)$

\begin{tabular}{lllllll}
\hline & $U^{11}$ & $U^{22}$ & $U^{33}$ & $U^{12}$ & $U^{13}$ & $U^{23}$ \\
\hline Au1 & $0.03350(16)$ & $0.02516(16)$ & $0.03426(18)$ & $0.00089(14)$ & $0.01226(13)$ & $-0.00147(14)$
\end{tabular}




\begin{tabular}{|c|c|c|c|c|c|c|}
\hline $\mathrm{Au} 2$ & $0.03692(18)$ & $0.03095(17)$ & $0.0360(2)$ & $0.00151(15)$ & $0.01285(14)$ & $-0.00129(14)$ \\
\hline Au3 & $0.03603(18)$ & $0.02896(17)$ & $0.0372(2)$ & $0.00002(14)$ & $0.01056(14)$ & -0.00228 \\
\hline P1 & $0.0365(10)$ & $0.0263(9)$ & $0.0337(11)$ & $0.0014(9)$ & $0.0129(9)$ & -0.0010 \\
\hline $\mathrm{P} 2$ & $0.0391(11)$ & $0.0341(11)$ & $0.0385(12)$ & $0.0042(9)$ & $0.0117(9)$ & $0.0010(9)$ \\
\hline P3 & $0.0416(12)$ & $0.0316(10)$ & $0.0361(12)$ & $-0.0012(9)$ & 0.0095 (9) & 0.0005 (9) \\
\hline O1 & 0.057 (4) & $0.045(4)$ & $0.053(4)$ & 0.007 (3) & $0.017(3)$ & $0.001(3)$ \\
\hline $\mathrm{O} 2$ & $0.045(3)$ & $0.041(3)$ & $0.037(3)$ & $0.000(3)$ & $0.006(3)$ & $-0.005(3)$ \\
\hline $\mathrm{O} 3$ & $0.043(3)$ & $0.045(3)$ & $0.038(4)$ & $0.001(3)$ & $0.005(3)$ & $0.001(3)$ \\
\hline $\mathrm{O} 4$ & $0.074(7)$ & $0.098(8)$ & $0.201(13)$ & $-0.002(6)$ & $0.070(8)$ & $0.033(8)$ \\
\hline $\mathrm{O5}$ & $0.078(6)$ & $0.059(5)$ & $0.070(5)$ & $0.008(4)$ & $0.006(4)$ & $0.012(4)$ \\
\hline O6 & $0.064(4)$ & $0.052(4)$ & $0.046(4)$ & $-0.008(3)$ & $0.025(3)$ & $-0.006(3)$ \\
\hline $\mathrm{O} 7$ & $0.061(4)$ & $0.056(4)$ & $0.057(4)$ & $0.002(4)$ & $0.028(4)$ & $0.008(4)$ \\
\hline $\mathrm{C} 1$ & 0.027 (4) & $0.028(4)$ & $0.025(4)$ & $-0.002(3)$ & $0.004(3)$ & $0.000(3)$ \\
\hline $\mathrm{C} 2$ & $0.040(5)$ & $0.035(5)$ & $0.043(5)$ & $-0.003(4)$ & $0.017(4)$ & $0.001(4)$ \\
\hline C3 & $0.041(5)$ & $0.027(4)$ & $0.033(5)$ & $-0.003(4)$ & 0.009 (4) & $0.000(3)$ \\
\hline $\mathrm{C} 4$ & $0.039(5)$ & $0.031(4)$ & $0.041(5)$ & $0.002(4)$ & $0.011(4)$ & $-0.001(4)$ \\
\hline C5 & $0.043(5)$ & $0.045(5)$ & $0.045(5)$ & $-0.011(4)$ & $0.017(4)$ & $0.000(4)$ \\
\hline C6 & $0.044(5)$ & $0.048(5)$ & $0.040(5)$ & $-0.010(4)$ & $0.013(4)$ & $0.003(4)$ \\
\hline $\mathrm{C} 7$ & $0.048(5)$ & $0.028(4)$ & $0.041(5)$ & $-0.003(4)$ & $0.005(4)$ & $-0.006(4)$ \\
\hline $\mathrm{C} 8$ & $0.035(4)$ & $0.030(4)$ & $0.032(4)$ & $-0.004(3)$ & $0.005(3)$ & $-0.007(3)$ \\
\hline C9 & $0.033(4)$ & $0.028(4)$ & $0.043(5)$ & $0.005(3)$ & $0.013(4)$ & $0.005(4)$ \\
\hline C10 & $0.044(5)$ & $0.018(4)$ & $0.042(5)$ & $0.002(3)$ & $0.013(4)$ & $0.002(3)$ \\
\hline C11 & $0.058(6)$ & $0.029(4)$ & $0.045(5)$ & $0.008(4)$ & $0.018(4)$ & $0.005(4)$ \\
\hline $\mathrm{C} 12$ & $0.046(5)$ & $0.032(4)$ & $0.069(7)$ & 0.009 (4) & $0.030(5)$ & $0.000(4)$ \\
\hline C13 & $0.034(5)$ & $0.038(5)$ & $0.059(6)$ & $0.003(4)$ & $0.006(4)$ & $0.001(4)$ \\
\hline $\mathrm{C} 14$ & $0.040(5)$ & $0.028(4)$ & $0.040(5)$ & $-0.002(4)$ & $0.006(4)$ & $0.001(4)$ \\
\hline $\mathrm{C} 15$ & $0.074(7)$ & $0.050(6)$ & $0.048(6)$ & $-0.006(6)$ & $0.009(5)$ & $-0.009(5)$ \\
\hline $\mathrm{C} 16$ & $0.051(6)$ & $0.062(6)$ & $0.050(6)$ & $-0.004(5)$ & $0.006(5)$ & $0.001(5)$ \\
\hline $\mathrm{C} 17$ & $0.044(5)$ & $0.029(4)$ & $0.035(5)$ & -0.005 & $0.017(4)$ & $-0.003(4)$ \\
\hline $\mathrm{C} 18$ & $0.060(6)$ & $0.040(5)$ & $0.039(5)$ & $0.004(4)$ & $0.023(4)$ & $-0.002(4)$ \\
\hline C19 & $0.081(8)$ & $0.047(6)$ & $0.047(6)$ & $-0.005(6)$ & $0.025(5)$ & $-0.018(5)$ \\
\hline $\mathrm{C} 20$ & $0.066(7)$ & $0.055(6)$ & $0.049(6)$ & -0.009 (6) & $0.012(5)$ & $-0.013(5)$ \\
\hline $\mathrm{C} 21$ & $0.055(6)$ & $0.068(7)$ & $0.047(6)$ & $-0.004(5)$ & $0.010(5)$ & $-0.008(5)$ \\
\hline $\mathrm{C} 22$ & $0.037(5)$ & $0.044(5)$ & $0.052(6)$ & $0.000(4)$ & $0.011(4)$ & $-0.006(4)$ \\
\hline $\mathrm{C} 23$ & $0.037(4)$ & $0.027(4)$ & $0.044(5)$ & $0.000(4)$ & $0.018(4)$ & $0.002(4)$ \\
\hline $\mathrm{C} 24$ & $0.055(5)$ & $0.026(4)$ & $0.042(5)$ & $-0.006(4)$ & $0.011(4)$ & $-0.002(4)$ \\
\hline $\mathrm{C} 25$ & $0.045(5)$ & $0.041(5)$ & $0.055(6)$ & $0.011(4)$ & $0.016(4)$ & $0.004(4)$ \\
\hline $\mathrm{C} 26$ & $0.036(5)$ & $0.054(5)$ & $0.041(5)$ & $0.006(4)$ & $0.013(4)$ & $0.001(4)$ \\
\hline $\mathrm{C} 27$ & $0.036(5)$ & $0.054(6)$ & $0.052(6)$ & $0.001(4)$ & 0.008 (4) & $0.009(5)$ \\
\hline $\mathrm{C} 28$ & $0.040(5)$ & $0.033(4)$ & $0.039(5)$ & $0.001(4)$ & $0.005(4)$ & 0.007 (4) \\
\hline $\mathrm{C} 29$ & $0.045(5)$ & $0.046(5)$ & $0.031(5)$ & $-0.001(4)$ & $0.010(4)$ & $0.000(4)$ \\
\hline $\mathrm{C} 30$ & $0.056(6)$ & $0.053(6)$ & $0.046(6)$ & $0.010(5)$ & $0.019(5)$ & $-0.007(5)$ \\
\hline $\mathrm{C} 31$ & $0.067(7)$ & $0.060(6)$ & $0.048(6)$ & $0.007(6)$ & $0.018(5)$ & $-0.001(5)$ \\
\hline $\mathrm{C} 32$ & $0.075(8)$ & $0.063(7)$ & $0.045(6)$ & $0.009(6)$ & $0.017(5)$ & $-0.002(5)$ \\
\hline $\mathrm{C} 33$ & $0.065(7)$ & $0.057(6)$ & $0.056(7)$ & $0.016(5)$ & $0.024(5)$ & $-0.007(5)$ \\
\hline $\mathrm{C} 34$ & $0.058(6)$ & $0.039(5)$ & $0.054(6)$ & $0.006(5)$ & $0.024(5)$ & $-0.004(5)$ \\
\hline $\mathrm{C} 35$ & $0.051(6)$ & $0.068(7)$ & $0.041(6)$ & $0.018(5)$ & $0.019(4)$ & $-0.011(5)$ \\
\hline $\mathrm{C} 36$ & $0.050(6)$ & $0.074(8)$ & $0.095(10)$ & $0.003(6)$ & $0.031(6)$ & $-0.007(7)$ \\
\hline
\end{tabular}




\begin{tabular}{|c|c|c|c|c|c|c|}
\hline C37 & $0.057(8)$ & 0.099 (11) & $0.121(13)$ & $-0.007(8)$ & $0.035(8)$ & $-0.021(10)$ \\
\hline C38 & $0.046(7)$ & $0.124(12)$ & $0.076(9)$ & $0.019(8)$ & $0.017(6)$ & $-0.031(9)$ \\
\hline $\mathrm{C} 39$ & $0.048(6)$ & $0.084(8)$ & $0.055(7)$ & $0.019(6)$ & $0.022(5)$ & $-0.011(6)$ \\
\hline $\mathrm{C} 40$ & $0.044(6)$ & $0.082(8)$ & $0.051(6)$ & $0.011(6)$ & $0.015(5)$ & $-0.018(6)$ \\
\hline $\mathrm{C} 41$ & $0.16(2)$ & $0.23(3)$ & $0.18(2)$ & 0.042 (19) & 0.101 (19) & $0.11(2)$ \\
\hline $\mathrm{C} 42$ & $0.099(11)$ & 0.094 (11) & $0.114(13)$ & $0.007(9)$ & $0.001(10)$ & $0.042(10)$ \\
\hline $\mathrm{C} 43$ & $0.045(5)$ & $0.029(4)$ & $0.057(6)$ & $0.002(4)$ & 0.015 & -0.001 \\
\hline $\mathrm{C} 44$ & $0.043(5)$ & $0.046(5)$ & $0.048(6)$ & $0.000(4)$ & 0.015 & $0.002(4)$ \\
\hline $\mathrm{C} 45$ & $0.067(7)$ & $0.046(6)$ & $0.049(6)$ & $-0.006(5)$ & $0.019(5)$ & $0.004(5)$ \\
\hline $\mathrm{C} 46$ & $0.083(8)$ & $0.030(5)$ & $0.061(7)$ & $0.000(5)$ & $0.014(6)$ & $0.003(5)$ \\
\hline $\mathrm{C} 47$ & $0.077(7)$ & $0.035(5)$ & $0.059(7)$ & $0.004(5)$ & $0.027(6)$ & $-0.003(5)$ \\
\hline $\mathrm{C} 48$ & $0.068(7)$ & $0.032(5)$ & $0.052(6)$ & $0.000(5)$ & $0.030(5)$ & $0.004(4)$ \\
\hline $\mathrm{C} 49$ & $0.048(5)$ & $0.040(5)$ & $0.043(5)$ & -0.001 & $0.021(4)$ & -0.007 (4) \\
\hline $\mathrm{C} 50$ & $0.039(5)$ & $0.051(6)$ & $0.067(7)$ & $0.006(5)$ & $0.018(5)$ & $0.008(5)$ \\
\hline C51 & $0.037(5)$ & $0.064(7)$ & $0.081(8)$ & $-0.001(5)$ & $0.013(5)$ & $0.003(6)$ \\
\hline C52 & $0.047(6)$ & $0.055(6)$ & $0.066(7)$ & $-0.023(5)$ & $0.017(5)$ & $-0.005(5)$ \\
\hline C53 & $0.058(6)$ & $0.040(5)$ & $0.072(7)$ & $-0.011(5)$ & $0.019(5)$ & $-0.007(5)$ \\
\hline C54 & $0.052(6)$ & $0.036(5)$ & $0.060(7)$ & -0.003 & $0.016(5)$ & $0.000(4)$ \\
\hline C55 & $0.040(5)$ & $0.036(5)$ & $0.039(5)$ & $-0.002(4)$ & $0.012(4)$ & $0.001(4)$ \\
\hline C56 & $0.045(5)$ & $0.036(5)$ & $0.048(6)$ & -0.004 & $0.014(4)$ & -0.001 \\
\hline C57 & $0.047(5)$ & $0.045(5)$ & $0.054(6)$ & $-0.006(4)$ & $0.016(5)$ & -0.007 (4) \\
\hline C58 & $0.050(6)$ & $0.062(6)$ & $0.045(6)$ & $-0.011(5)$ & $0.013(5)$ & $-0.015(5)$ \\
\hline C59 & $0.036(5)$ & $0.063(6)$ & $0.052(6)$ & $0.004(5)$ & 0.009 (4) & $-0.001(5)$ \\
\hline C60 & $0.043(5)$ & $0.039(5)$ & $0.036(5)$ & -0.003 & $0.018(4)$ & $0.001(4)$ \\
\hline C61 & $0.031(4)$ & $0.047(5)$ & $0.042(5)$ & -0.002 & $0.009(4)$ & -0.003 \\
\hline C62 & $0.033(4)$ & $0.045(5)$ & $0.046(5)$ & -0.002 & $0.013(4)$ & -0.001 \\
\hline C63 & $0.052(6)$ & $0.046(5)$ & $0.061(7)$ & $0.000(5)$ & $0.020(5)$ & $-0.002(5)$ \\
\hline C64 & $0.051(6)$ & $0.041(5)$ & $0.056(6)$ & $0.013(4)$ & $0.009(5)$ & $-0.005(5)$ \\
\hline C65 & $0.043(5)$ & $0.035(5)$ & $0.073(7)$ & $0.004(4)$ & $0.010(5)$ & $0.009(5)$ \\
\hline C66 & 0.040 & $0.041(5)$ & $0.051(6)$ & -0.002 & $0.014(4)$ & $0.012(4)$ \\
\hline C67 & $0.091(9)$ & $0.073(8)$ & $0.054(7)$ & $-0.031(7)$ & $0.032(6)$ & $-0.002(6)$ \\
\hline C68 & $0.081(8)$ & $0.070(8)$ & $0.062(7)$ & $0.005(6)$ & $0.036(6)$ & $0.016(6)$ \\
\hline C69 & $0.043(5)$ & $0.025(4)$ & $0.055(6)$ & 0.005 & $0.011(4)$ & $-0.006(4)$ \\
\hline $\mathrm{C} 70$ & $0.040(5)$ & $0.047(6)$ & $0.075(8)$ & $0.008(5)$ & $0.005(5)$ & $0.000(5)$ \\
\hline C71 & $0.042(6)$ & $0.028(5)$ & $0.147(13)$ & $0.004(4)$ & $0.031(7)$ & $0.015(6)$ \\
\hline $\mathrm{C} 72$ & $0.083(9)$ & $0.036(5)$ & $0.111(11)$ & $-0.010(6)$ & $0.059(8)$ & $-0.026(6)$ \\
\hline $\mathrm{C} 73$ & $0.074(8)$ & $0.048(6)$ & $0.099(10)$ & $-0.021(6)$ & $0.048(7)$ & $-0.026(6)$ \\
\hline $\mathrm{C} 74$ & $0.051(6)$ & $0.043(5)$ & $0.051(6)$ & $0.000(4)$ & $0.015(5)$ & -0.006 \\
\hline C75 & $0.063(7)$ & $0.057(6)$ & $0.049(6)$ & $-0.012(5)$ & 0.007 (5) & $0.011(5)$ \\
\hline C76 & $0.095(9)$ & $0.035(5)$ & $0.060(7)$ & $-0.001(5)$ & $0.033(6)$ & $0.011(5)$ \\
\hline C77 & $0.164(17)$ & $0.055(8)$ & $0.088(11)$ & $0.003(9)$ & $0.045(11)$ & $0.026(7)$ \\
\hline $\mathrm{C} 78$ & $0.24(2)$ & $0.061(9)$ & $0.136(15)$ & $0.039(12)$ & $0.134(17)$ & $0.041(9)$ \\
\hline C79 & $0.173(17)$ & $0.110(12)$ & $0.054(8)$ & $0.010(12)$ & $0.068(10)$ & $0.011(8)$ \\
\hline $\mathrm{C} 80$ & $0.28(3)$ & $0.047(7)$ & $0.090(11)$ & $0.004(11)$ & $0.108(14)$ & $0.000(7)$ \\
\hline S1A & $0.0655(17)$ & $0.0536(15)$ & 0.0668 (19) & $0.0006(13)$ & $0.0234(14)$ & $-0.0011(13)$ \\
\hline $\mathrm{S} 2 \mathrm{~A}$ & $0.0580(16)$ & $0.0746(18)$ & $0.0583(17)$ & $0.0027(15)$ & $0.0125(13)$ & $-0.0114(15)$ \\
\hline F1A & $0.091(6)$ & $0.132(7)$ & $0.092(6)$ & $-0.038(5)$ & $-0.019(5)$ & $0.025(5)$ \\
\hline $\mathrm{F} 2 \mathrm{~A}$ & $0.175(9)$ & $0.061(4)$ & $0.113(7)$ & $-0.006(5)$ & $0.070(7)$ & $0.000(5)$ \\
\hline
\end{tabular}




\begin{tabular}{lllllll} 
F3A & $0.100(6)$ & $0.092(5)$ & $0.071(5)$ & $0.003(5)$ & $0.032(4)$ & $0.013(4)$ \\
F4A & $0.075(5)$ & $0.094(5)$ & $0.070(5)$ & $0.024(4)$ & $0.008(4)$ & $-0.002(4)$ \\
F5A & $0.108(6)$ & $0.103(6)$ & $0.063(5)$ & $0.015(5)$ & $0.018(4)$ & $-0.026(4)$ \\
F6A & $0.114(7)$ & $0.091(6)$ & $0.095(6)$ & $-0.014(5)$ & $0.055(5)$ & $0.002(5)$ \\
O1A & $0.068(6)$ & $0.091(7)$ & $0.125(9)$ & $0.025(5)$ & $0.014(6)$ & $-0.010(6)$ \\
O2A & $0.144(9)$ & $0.073(6)$ & $0.073(6)$ & $-0.010(6)$ & $0.055(6)$ & $-0.019(5)$ \\
O3A & $0.082(6)$ & $0.110(7)$ & $0.056(5)$ & $0.033(5)$ & $-0.005(4)$ & $-0.008(5)$ \\
O4A & $0.115(8)$ & $0.065(5)$ & $0.086(6)$ & $-0.025(5)$ & $0.049(6)$ & $-0.018(5)$ \\
N1A & $0.060(6)$ & $0.064(6)$ & $0.068(6)$ & $-0.004(5)$ & $0.007(5)$ & $-0.014(5)$ \\
C1A & $0.075(8)$ & $0.067(7)$ & $0.060(8)$ & $-0.001(7)$ & $0.008(6)$ & $-0.001(6)$ \\
C2A & $0.089(9)$ & $0.074(8)$ & $0.048(7)$ & $0.007(7)$ & $0.000(6)$ & $0.004(6)$ \\
\hline
\end{tabular}

Geometric parameters $\left(\hat{A},{ }^{o}\right)$

\begin{tabular}{|c|c|c|c|}
\hline $\mathrm{Au} 1-\mathrm{Au} 2$ & $3.3005(5)$ & $\mathrm{C} 41-\mathrm{H} 41 \mathrm{~B}$ & 0.9800 \\
\hline $\mathrm{Au} 1-\mathrm{Au} 3$ & $3.1909(5)$ & $\mathrm{C} 41-\mathrm{H} 41 \mathrm{C}$ & 0.9800 \\
\hline $\mathrm{Au} 1-\mathrm{P} 1$ & $2.280(2)$ & $\mathrm{C} 42-\mathrm{H} 42 \mathrm{~A}$ & 0.9800 \\
\hline $\mathrm{Au} 1-\mathrm{C} 1$ & $2.094(8)$ & $\mathrm{C} 42-\mathrm{H} 42 \mathrm{~B}$ & 0.9800 \\
\hline $\mathrm{Au} 2-\mathrm{Au} 3$ & $3.2101(5)$ & $\mathrm{C} 42-\mathrm{H} 42 \mathrm{C}$ & 0.9800 \\
\hline $\mathrm{Au} 2-\mathrm{P} 2$ & $2.281(2)$ & $\mathrm{C} 43-\mathrm{H} 43$ & 1.0000 \\
\hline $\mathrm{Au} 2-\mathrm{C} 1$ & $2.098(7)$ & $\mathrm{C} 43-\mathrm{C} 44$ & $1.539(12)$ \\
\hline $\mathrm{Au} 3-\mathrm{P} 3$ & $2.273(2)$ & $\mathrm{C} 43-\mathrm{C} 48$ & $1.536(13)$ \\
\hline $\mathrm{Au} 3-\mathrm{C} 1$ & $2.090(7)$ & $\mathrm{C} 44-\mathrm{H} 44 \mathrm{~A}$ & 0.9900 \\
\hline $\mathrm{P} 1-\mathrm{C} 3$ & $1.840(8)$ & $\mathrm{C} 44-\mathrm{H} 44 \mathrm{~B}$ & 0.9900 \\
\hline $\mathrm{P} 1-\mathrm{C} 17$ & $1.846(8)$ & $\mathrm{C} 44-\mathrm{C} 45$ & $1.520(13)$ \\
\hline $\mathrm{P} 1-\mathrm{C} 23$ & $1.837(8)$ & $\mathrm{C} 45-\mathrm{H} 45 \mathrm{~A}$ & 0.9900 \\
\hline $\mathrm{P} 2-\mathrm{C} 29$ & $1.833(9)$ & $\mathrm{C} 45-\mathrm{H} 45 \mathrm{~B}$ & 0.9900 \\
\hline $\mathrm{P} 2-\mathrm{C} 43$ & $1.852(8)$ & $\mathrm{C} 45-\mathrm{C} 46$ & $1.516(14)$ \\
\hline $\mathrm{P} 2-\mathrm{C} 49$ & $1.853(9)$ & $\mathrm{C} 46-\mathrm{H} 46 \mathrm{~A}$ & 0.9900 \\
\hline $\mathrm{P} 3-\mathrm{C} 55$ & $1.835(9)$ & $\mathrm{C} 46-\mathrm{H} 46 \mathrm{~B}$ & 0.9900 \\
\hline P3-C69 & $1.854(9)$ & $\mathrm{C} 46-\mathrm{C} 47$ & $1.526(14)$ \\
\hline $\mathrm{P} 3-\mathrm{C} 75$ & $1.847(10)$ & $\mathrm{C} 47-\mathrm{H} 47 \mathrm{~A}$ & 0.9900 \\
\hline $\mathrm{O} 1-\mathrm{C} 2$ & $1.176(10)$ & $\mathrm{C} 47-\mathrm{H} 47 \mathrm{~B}$ & 0.9900 \\
\hline $\mathrm{O} 2-\mathrm{C} 10$ & $1.366(10)$ & $\mathrm{C} 47-\mathrm{C} 48$ & $1.534(12)$ \\
\hline $\mathrm{O} 2-\mathrm{C} 15$ & $1.427(11)$ & $\mathrm{C} 48-\mathrm{H} 48 \mathrm{~A}$ & 0.9900 \\
\hline $\mathrm{O} 3-\mathrm{C} 14$ & $1.368(10)$ & $\mathrm{C} 48-\mathrm{H} 48 \mathrm{~B}$ & 0.9900 \\
\hline $\mathrm{O} 3-\mathrm{C} 16$ & $1.441(11)$ & C49-H49 & 1.0000 \\
\hline $\mathrm{O} 4-\mathrm{C} 36$ & $1.397(16)$ & $\mathrm{C} 49-\mathrm{C} 50$ & $1.546(13)$ \\
\hline $\mathrm{O} 4-\mathrm{C} 41$ & $1.278(18)$ & $\mathrm{C} 49-\mathrm{C} 54$ & $1.523(12)$ \\
\hline $\mathrm{O} 5-\mathrm{C} 40$ & $1.360(13)$ & $\mathrm{C} 50-\mathrm{H} 50 \mathrm{~A}$ & 0.9900 \\
\hline $\mathrm{O} 5-\mathrm{C} 42$ & $1.438(14)$ & С $50-\mathrm{H} 50 \mathrm{~B}$ & 0.9900 \\
\hline $\mathrm{O} 6-\mathrm{C} 62$ & $1.376(11)$ & $\mathrm{C} 50-\mathrm{C} 51$ & $1.541(14)$ \\
\hline O6- $-\mathrm{C} 67$ & $1.432(12)$ & C51-H51A & 0.9900 \\
\hline $\mathrm{O} 7-\mathrm{C} 66$ & $1.378(11)$ & С51-H51B & 0.9900 \\
\hline $\mathrm{O} 7-\mathrm{C} 68$ & $1.422(12)$ & $\mathrm{C} 51-\mathrm{C} 52$ & $1.503(14)$ \\
\hline $\mathrm{C} 1-\mathrm{C} 2$ & $1.282(12)$ & $\mathrm{C} 52-\mathrm{H} 52 \mathrm{~A}$ & 0.9900 \\
\hline $\mathrm{C} 3-\mathrm{C} 4$ & $1.385(11)$ & С $52-\mathrm{H} 52 \mathrm{~B}$ & 0.9900 \\
\hline $\mathrm{C} 3-\mathrm{C} 8$ & $1.409(11)$ & $\mathrm{C} 52-\mathrm{C} 53$ & $1.524(14)$ \\
\hline
\end{tabular}




\begin{tabular}{|c|c|c|c|}
\hline $\mathrm{C} 4-\mathrm{H} 4$ & 0.9500 & $\mathrm{C} 53-\mathrm{H} 53 \mathrm{~A}$ & 0.9900 \\
\hline $\mathrm{C} 4-\mathrm{C} 5$ & $1.373(12)$ & $\mathrm{C} 53-\mathrm{H} 53 \mathrm{~B}$ & 0.9900 \\
\hline $\mathrm{C} 5-\mathrm{H} 5$ & 0.9500 & $\mathrm{C} 53-\mathrm{C} 54$ & $1.537(13)$ \\
\hline $\mathrm{C} 5-\mathrm{C} 6$ & $1.364(13)$ & $\mathrm{C} 54-\mathrm{H} 54 \mathrm{~A}$ & 0.9900 \\
\hline $\mathrm{C} 6-\mathrm{H} 6$ & 0.9500 & C54-H54B & 0.9900 \\
\hline $\mathrm{C} 6-\mathrm{C} 7$ & $1.394(12)$ & $\mathrm{C} 55-\mathrm{C} 56$ & $1.395(12)$ \\
\hline $\mathrm{C} 7-\mathrm{H} 7$ & 0.9500 & $\mathrm{C} 55-\mathrm{C} 60$ & $1.412(12)$ \\
\hline $\mathrm{C} 7-\mathrm{C} 8$ & $1.394(12)$ & C56-H56 & 0.9500 \\
\hline $\mathrm{C} 8-\mathrm{C} 9$ & $1.486(11)$ & $\mathrm{C} 56-\mathrm{C} 57$ & $1.383(13)$ \\
\hline $\mathrm{C} 9-\mathrm{C} 10$ & $1.401(12)$ & C57-H57 & 0.9500 \\
\hline $\mathrm{C} 9-\mathrm{C} 14$ & $1.389(11)$ & $\mathrm{C} 57-\mathrm{C} 58$ & $1.359(14)$ \\
\hline $\mathrm{C} 10-\mathrm{C} 11$ & $1.390(12)$ & C58-H58 & 0.9500 \\
\hline $\mathrm{C} 11-\mathrm{H} 11$ & 0.9500 & $\mathrm{C} 58-\mathrm{C} 59$ & $1.395(14)$ \\
\hline $\mathrm{C} 11-\mathrm{C} 12$ & $1.374(13)$ & C59-H59 & 0.9500 \\
\hline $\mathrm{C} 12-\mathrm{H} 12$ & 0.9500 & $\mathrm{C} 59-\mathrm{C} 60$ & $1.375(12)$ \\
\hline $\mathrm{C} 12-\mathrm{C} 13$ & $1.365(13)$ & $\mathrm{C} 60-\mathrm{C} 61$ & $1.499(12)$ \\
\hline $\mathrm{C} 13-\mathrm{H} 13$ & 0.9500 & C61-C62 & $1.390(12)$ \\
\hline $\mathrm{C} 13-\mathrm{C} 14$ & $1.390(12)$ & C61-C66 & $1.404(12)$ \\
\hline C15-H15A & 0.9800 & $\mathrm{C} 62-\mathrm{C} 63$ & $1.386(13)$ \\
\hline C15-H15B & 0.9800 & C63-H63 & 0.9500 \\
\hline $\mathrm{C} 15-\mathrm{H} 15 \mathrm{C}$ & 0.9800 & $\mathrm{C} 63-\mathrm{C} 64$ & $1.372(14)$ \\
\hline $\mathrm{C} 16-\mathrm{H} 16 \mathrm{~A}$ & 0.9800 & C64-H64 & 0.9500 \\
\hline C16-H16B & 0.9800 & $\mathrm{C} 64-\mathrm{C} 65$ & $1.372(14)$ \\
\hline $\mathrm{C} 16-\mathrm{H} 16 \mathrm{C}$ & 0.9800 & C65-H65 & 0.9500 \\
\hline $\mathrm{C} 17-\mathrm{H} 17$ & 1.0000 & $\mathrm{C} 65-\mathrm{C} 66$ & $1.374(13)$ \\
\hline $\mathrm{C} 17-\mathrm{C} 18$ & $1.533(11)$ & C67-H67A & 0.9800 \\
\hline $\mathrm{C} 17-\mathrm{C} 22$ & $1.527(12)$ & C67-H67B & 0.9800 \\
\hline $\mathrm{C} 18-\mathrm{H} 18 \mathrm{~A}$ & 0.9900 & C67-H67C & 0.9800 \\
\hline $\mathrm{C} 18-\mathrm{H} 18 \mathrm{~B}$ & 0.9900 & C68-H68A & 0.9800 \\
\hline $\mathrm{C} 18-\mathrm{C} 19$ & $1.523(13)$ & C68-H68B & 0.9800 \\
\hline C19-H19A & 0.9900 & $\mathrm{C} 68-\mathrm{H} 68 \mathrm{C}$ & 0.9800 \\
\hline С19-H19B & 0.9900 & C69-H69 & 1.0000 \\
\hline $\mathrm{C} 19-\mathrm{C} 20$ & $1.522(15)$ & $\mathrm{C} 69-\mathrm{C} 70$ & $1.529(12)$ \\
\hline $\mathrm{C} 20-\mathrm{H} 20 \mathrm{~A}$ & 0.9900 & $\mathrm{C} 69-\mathrm{C} 74$ & $1.530(13)$ \\
\hline $\mathrm{C} 20-\mathrm{H} 20 \mathrm{~B}$ & 0.9900 & $\mathrm{C} 70-\mathrm{H} 70 \mathrm{~A}$ & 0.9900 \\
\hline $\mathrm{C} 20-\mathrm{C} 21$ & $1.520(14)$ & $\mathrm{C} 70-\mathrm{H} 70 \mathrm{~B}$ & 0.9900 \\
\hline $\mathrm{C} 21-\mathrm{H} 21 \mathrm{~A}$ & 0.9900 & $\mathrm{C} 70-\mathrm{C} 71$ & $1.539(14)$ \\
\hline $\mathrm{C} 21-\mathrm{H} 21 \mathrm{~B}$ & 0.9900 & $\mathrm{C} 71-\mathrm{H} 71 \mathrm{~A}$ & 0.9900 \\
\hline $\mathrm{C} 21-\mathrm{C} 22$ & $1.535(13)$ & $\mathrm{C} 71-\mathrm{H} 71 \mathrm{~B}$ & 0.9900 \\
\hline $\mathrm{C} 22-\mathrm{H} 22 \mathrm{~A}$ & 0.9900 & $\mathrm{C} 71-\mathrm{C} 72$ & $1.520(17)$ \\
\hline $\mathrm{C} 22-\mathrm{H} 22 \mathrm{~B}$ & 0.9900 & $\mathrm{C} 72-\mathrm{H} 72 \mathrm{~A}$ & 0.9900 \\
\hline $\mathrm{C} 23-\mathrm{H} 23$ & 1.0000 & $\mathrm{C} 72-\mathrm{H} 72 \mathrm{~B}$ & 0.9900 \\
\hline $\mathrm{C} 23-\mathrm{C} 24$ & $1.551(11)$ & $\mathrm{C} 72-\mathrm{C} 73$ & $1.504(17)$ \\
\hline $\mathrm{C} 23-\mathrm{C} 28$ & $1.531(11)$ & $\mathrm{C} 73-\mathrm{H} 73 \mathrm{~A}$ & 0.9900 \\
\hline $\mathrm{C} 24-\mathrm{H} 24 \mathrm{~A}$ & 0.9900 & $\mathrm{C} 73-\mathrm{H} 73 \mathrm{~B}$ & 0.9900 \\
\hline $\mathrm{C} 24-\mathrm{H} 24 \mathrm{~B}$ & 0.9900 & $\mathrm{C} 73-\mathrm{C} 74$ & $1.524(13)$ \\
\hline $\mathrm{C} 24-\mathrm{C} 25$ & $1.541(12)$ & $\mathrm{C} 74-\mathrm{H} 74 \mathrm{~A}$ & 0.9900 \\
\hline $\mathrm{C} 25-\mathrm{H} 25 \mathrm{~A}$ & 0.9900 & C74-H74B & 0.9900 \\
\hline
\end{tabular}




\begin{tabular}{|c|c|c|c|}
\hline $\mathrm{C} 25-\mathrm{H} 25 \mathrm{~B}$ & 0.9900 & $\mathrm{C} 75-\mathrm{H} 75$ & 1.0000 \\
\hline $\mathrm{C} 25-\mathrm{C} 26$ & $1.533(13)$ & $\mathrm{C} 75-\mathrm{C} 76$ & $1.497(14)$ \\
\hline $\mathrm{C} 26-\mathrm{H} 26 \mathrm{~A}$ & 0.9900 & $\mathrm{C} 75-\mathrm{C} 80$ & $1.444(16)$ \\
\hline $\mathrm{C} 26-\mathrm{H} 26 \mathrm{~B}$ & 0.9900 & $\mathrm{C} 76-\mathrm{H} 76 \mathrm{~A}$ & 0.9900 \\
\hline $\mathrm{C} 26-\mathrm{C} 27$ & $1.529(13)$ & $\mathrm{C} 76-\mathrm{H} 76 \mathrm{~B}$ & 0.9900 \\
\hline $\mathrm{C} 27-\mathrm{H} 27 \mathrm{~A}$ & 0.9900 & $\mathrm{C} 76-\mathrm{C} 77$ & $1.498(15)$ \\
\hline $\mathrm{C} 27-\mathrm{H} 27 \mathrm{~B}$ & 0.9900 & C77-H77A & 0.9900 \\
\hline $\mathrm{C} 27-\mathrm{C} 28$ & $1.524(11)$ & C77-H77B & 0.9900 \\
\hline $\mathrm{C} 28-\mathrm{H} 28 \mathrm{~A}$ & 0.9900 & $\mathrm{C} 77-\mathrm{C} 78$ & $1.416(19)$ \\
\hline $\mathrm{C} 28-\mathrm{H} 28 \mathrm{~B}$ & 0.9900 & $\mathrm{C} 78-\mathrm{H} 78 \mathrm{~A}$ & 0.9900 \\
\hline $\mathrm{C} 29-\mathrm{C} 30$ & $1.421(13)$ & $\mathrm{C} 78-\mathrm{H} 78 \mathrm{~B}$ & 0.9900 \\
\hline $\mathrm{C} 29-\mathrm{C} 34$ & $1.389(13)$ & $\mathrm{C} 78-\mathrm{C} 79$ & $1.56(2)$ \\
\hline $\mathrm{C} 30-\mathrm{H} 30$ & 0.9500 & $\mathrm{C} 79-\mathrm{H} 79 \mathrm{~A}$ & 0.9900 \\
\hline $\mathrm{C} 30-\mathrm{C} 31$ & $1.376(14)$ & C79-H79B & 0.9900 \\
\hline $\mathrm{C} 31-\mathrm{H} 31$ & 0.9500 & $\mathrm{C} 79-\mathrm{C} 80$ & $1.539(18)$ \\
\hline $\mathrm{C} 31-\mathrm{C} 32$ & $1.391(14)$ & $\mathrm{C} 80-\mathrm{H} 80 \mathrm{~A}$ & 0.9900 \\
\hline C $32-\mathrm{H} 32$ & 0.9500 & $\mathrm{C} 80-\mathrm{H} 80 \mathrm{~B}$ & 0.9900 \\
\hline $\mathrm{C} 32-\mathrm{C} 33$ & $1.368(15)$ & $\mathrm{S} 1 \mathrm{~A}-\mathrm{O} 1 \mathrm{~A}$ & $1.450(9)$ \\
\hline $\mathrm{C} 33-\mathrm{H} 33$ & 0.9500 & $\mathrm{~S} 1 \mathrm{~A}-\mathrm{O} 2 \mathrm{~A}$ & $1.436(9)$ \\
\hline C33-C34 & $1.383(14)$ & $\mathrm{S} 1 \mathrm{~A}-\mathrm{N} 1 \mathrm{~A}$ & $1.570(10)$ \\
\hline $\mathrm{C} 34-\mathrm{C} 35$ & $1.496(14)$ & $\mathrm{S} 1 \mathrm{~A}-\mathrm{C} 1 \mathrm{~A}$ & $1.784(13)$ \\
\hline $\mathrm{C} 35-\mathrm{C} 36$ & $1.366(16)$ & $\mathrm{S} 2 \mathrm{~A}-\mathrm{O} 3 \mathrm{~A}$ & $1.448(8)$ \\
\hline $\mathrm{C} 35-\mathrm{C} 40$ & $1.376(16)$ & $\mathrm{S} 2 \mathrm{~A}-\mathrm{O} 4 \mathrm{~A}$ & $1.453(9)$ \\
\hline $\mathrm{C} 36-\mathrm{C} 37$ & $1.376(18)$ & $\mathrm{S} 2 \mathrm{~A}-\mathrm{N} 1 \mathrm{~A}$ & $1.566(9)$ \\
\hline C37-H37 & 0.9500 & $\mathrm{~S} 2 \mathrm{~A}-\mathrm{C} 2 \mathrm{~A}$ & $1.754(14)$ \\
\hline $\mathrm{C} 37-\mathrm{C} 38$ & $1.40(2)$ & $\mathrm{F} 1 \mathrm{~A}-\mathrm{C} 1 \mathrm{~A}$ & $1.321(14)$ \\
\hline $\mathrm{C} 38-\mathrm{H} 38$ & 0.9500 & $\mathrm{~F} 2 \mathrm{~A}-\mathrm{C} 1 \mathrm{~A}$ & $1.342(14)$ \\
\hline $\mathrm{C} 38-\mathrm{C} 39$ & $1.356(17)$ & $\mathrm{F} 3 \mathrm{~A}-\mathrm{C} 1 \mathrm{~A}$ & $1.346(14)$ \\
\hline C39-H39 & 0.9500 & $\mathrm{~F} 4 \mathrm{~A}-\mathrm{C} 2 \mathrm{~A}$ & $1.301(13)$ \\
\hline $\mathrm{C} 39-\mathrm{C} 40$ & $1.386(14)$ & $\mathrm{F} 5 \mathrm{~A}-\mathrm{C} 2 \mathrm{~A}$ & $1.360(14)$ \\
\hline $\mathrm{C} 41-\mathrm{H} 41 \mathrm{~A}$ & 0.9800 & $\mathrm{~F} 6 \mathrm{~A}-\mathrm{C} 2 \mathrm{~A}$ & $1.368(14)$ \\
\hline $\mathrm{Au} 3-\mathrm{Au} 1-\mathrm{Au} 2$ & $59.247(11)$ & $\mathrm{P} 2-\mathrm{C} 43-\mathrm{H} 43$ & 108.8 \\
\hline $\mathrm{P} 1-\mathrm{Au} 1-\mathrm{Au} 2$ & $138.37(5)$ & $\mathrm{C} 44-\mathrm{C} 43-\mathrm{P} 2$ & $109.0(6)$ \\
\hline P1-Au1-Au3 & $133.55(5)$ & $\mathrm{C} 44-\mathrm{C} 43-\mathrm{H} 43$ & 108.8 \\
\hline $\mathrm{C} 1-\mathrm{Au} 1-\mathrm{Au} 2$ & $38.1(2)$ & $\mathrm{C} 48-\mathrm{C} 43-\mathrm{P} 2$ & $111.4(6)$ \\
\hline $\mathrm{C} 1-\mathrm{Au} 1-\mathrm{Au} 3$ & $40.3(2)$ & $\mathrm{C} 48-\mathrm{C} 43-\mathrm{H} 43$ & 108.8 \\
\hline $\mathrm{C} 1-\mathrm{Au} 1-\mathrm{P} 1$ & $172.5(2)$ & $\mathrm{C} 48-\mathrm{C} 43-\mathrm{C} 44$ & $110.0(8)$ \\
\hline $\mathrm{Au} 3-\mathrm{Au} 2-\mathrm{Au} 1$ & $58.675(11)$ & $\mathrm{C} 43-\mathrm{C} 44-\mathrm{H} 44 \mathrm{~A}$ & 109.4 \\
\hline $\mathrm{P} 2-\mathrm{Au} 2-\mathrm{Au} 1$ & $132.86(6)$ & $\mathrm{C} 43-\mathrm{C} 44-\mathrm{H} 44 \mathrm{~B}$ & 109.4 \\
\hline $\mathrm{P} 2-\mathrm{Au} 2-\mathrm{Au} 3$ & $141.89(6)$ & $\mathrm{H} 44 \mathrm{~A}-\mathrm{C} 44-\mathrm{H} 44 \mathrm{~B}$ & 108.0 \\
\hline $\mathrm{C} 1-\mathrm{Au} 2-\mathrm{Au} 1$ & $38.0(2)$ & $\mathrm{C} 45-\mathrm{C} 44-\mathrm{C} 43$ & $111.0(8)$ \\
\hline $\mathrm{C} 1-\mathrm{Au} 2-\mathrm{Au} 3$ & $39.9(2)$ & $\mathrm{C} 45-\mathrm{C} 44-\mathrm{H} 44 \mathrm{~A}$ & 109.4 \\
\hline $\mathrm{C} 1-\mathrm{Au} 2-\mathrm{P} 2$ & $170.9(2)$ & $\mathrm{C} 45-\mathrm{C} 44-\mathrm{H} 44 \mathrm{~B}$ & 109.4 \\
\hline $\mathrm{Au} 1-\mathrm{Au} 3-\mathrm{Au} 2$ & $62.077(11)$ & $\mathrm{C} 44-\mathrm{C} 45-\mathrm{H} 45 \mathrm{~A}$ & 109.4 \\
\hline P3-Au3-Au1 & $136.88(6)$ & $\mathrm{C} 44-\mathrm{C} 45-\mathrm{H} 45 \mathrm{~B}$ & 109.4 \\
\hline $\mathrm{P} 3-\mathrm{Au} 3-\mathrm{Au} 2$ & $134.46(6)$ & $\mathrm{H} 45 \mathrm{~A}-\mathrm{C} 45-\mathrm{H} 45 \mathrm{~B}$ & 108.0 \\
\hline $\mathrm{C} 1-\mathrm{Au} 3-\mathrm{Au} 1$ & $40.3(2)$ & $\mathrm{C} 46-\mathrm{C} 45-\mathrm{C} 44$ & $111.4(8)$ \\
\hline
\end{tabular}




\begin{tabular}{|c|c|}
\hline $\mathrm{C} 1-\mathrm{Au} 3-\mathrm{Au} 2$ & $40.1(2)$ \\
\hline $\mathrm{C} 1-\mathrm{Au} 3-\mathrm{P} 3$ & $173.3(2)$ \\
\hline $\mathrm{C} 3-\mathrm{P} 1-\mathrm{Au} 1$ & $122.2(3)$ \\
\hline $\mathrm{C} 3-\mathrm{P} 1-\mathrm{C} 17$ & $104.8(4)$ \\
\hline $\mathrm{C} 17-\mathrm{P} 1-\mathrm{Au} 1$ & 108.5 \\
\hline $\mathrm{C} 23-\mathrm{P} 1-\mathrm{Au} 1$ & $110.1(3)$ \\
\hline $\mathrm{C} 23-\mathrm{P} 1-\mathrm{C} 3$ & $104.3(4)$ \\
\hline $\mathrm{C} 23-\mathrm{P} 1-\mathrm{C} 17$ & $105.8(4)$ \\
\hline $\mathrm{C} 29-\mathrm{P} 2-\mathrm{Au} 2$ & $121.8(3)$ \\
\hline $\mathrm{C} 29-\mathrm{P} 2-\mathrm{C} 43$ & $104.5(4)$ \\
\hline $\mathrm{C} 29-\mathrm{P} 2-\mathrm{C} 49$ & $104.8(4)$ \\
\hline $\mathrm{C} 43-\mathrm{P} 2-\mathrm{Au} 2$ & $112.2(3)$ \\
\hline $\mathrm{C} 43-\mathrm{P} 2-\mathrm{C} 49$ & $108.7(4)$ \\
\hline $\mathrm{C} 49-\mathrm{P} 2-\mathrm{Au} 2$ & $104.0(3)$ \\
\hline $\mathrm{C} 55-\mathrm{P} 3-\mathrm{Au} 3$ & $121.0(3)$ \\
\hline $\mathrm{C} 55-\mathrm{P} 3-\mathrm{C} 69$ & $104.2(4)$ \\
\hline $\mathrm{C} 55-\mathrm{P} 3-\mathrm{C} 75$ & $105.0(4)$ \\
\hline C69-P3-Au3 & $110.1(3)$ \\
\hline $\mathrm{C} 75-\mathrm{P} 3-\mathrm{Au} 3$ & $111.6(4)$ \\
\hline C75-P3-C69 & $103.4(5)$ \\
\hline $\mathrm{C} 10-\mathrm{O} 2-\mathrm{C} 15$ & $117.5(7)$ \\
\hline $\mathrm{C} 14-\mathrm{O} 3-\mathrm{C} 16$ & $117.1(7)$ \\
\hline $\mathrm{C} 41-\mathrm{O} 4-\mathrm{C} 36$ & $124.5(14)$ \\
\hline $\mathrm{C} 40-\mathrm{O} 5-\mathrm{C} 42$ & $116.7(10)$ \\
\hline C62-O6-C67 & $116.4(8)$ \\
\hline $\mathrm{C} 66-\mathrm{O} 7-\mathrm{C} 68$ & $116.7(8)$ \\
\hline $\mathrm{Au} 1-\mathrm{C} 1-\mathrm{Au} 2$ & $103.9(3)$ \\
\hline $\mathrm{Au} 3-\mathrm{C} 1-\mathrm{Au} 1$ & $99.4(3)$ \\
\hline $\mathrm{Au} 3-\mathrm{C} 1-\mathrm{Au} 2$ & $100.1(3)$ \\
\hline $\mathrm{C} 2-\mathrm{C} 1-\mathrm{Au} 1$ & $116.3(6)$ \\
\hline $\mathrm{C} 2-\mathrm{C} 1-\mathrm{Au} 2$ & $115.6(6)$ \\
\hline $\mathrm{C} 2-\mathrm{C} 1-\mathrm{Au} 3$ & $118.8(6)$ \\
\hline $\mathrm{O} 1-\mathrm{C} 2-\mathrm{C} 1$ & $178.9(10)$ \\
\hline $\mathrm{C} 4-\mathrm{C} 3-\mathrm{P} 1$ & $117.5(6)$ \\
\hline $\mathrm{C} 4-\mathrm{C} 3-\mathrm{C} 8$ & $118.2(8)$ \\
\hline $\mathrm{C} 8-\mathrm{C} 3-\mathrm{P} 1$ & $123.8(6)$ \\
\hline $\mathrm{C} 3-\mathrm{C} 4-\mathrm{H} 4$ & 118.6 \\
\hline $\mathrm{C} 5-\mathrm{C} 4-\mathrm{C} 3$ & $122.9(8)$ \\
\hline $\mathrm{C} 5-\mathrm{C} 4-\mathrm{H} 4$ & 118.6 \\
\hline $\mathrm{C} 4-\mathrm{C} 5-\mathrm{H} 5$ & 120.4 \\
\hline $\mathrm{C} 6-\mathrm{C} 5-\mathrm{C} 4$ & $119.3(9)$ \\
\hline $\mathrm{C} 6-\mathrm{C} 5-\mathrm{H} 5$ & 120.4 \\
\hline $\mathrm{C} 5-\mathrm{C} 6-\mathrm{H} 6$ & 120.1 \\
\hline $\mathrm{C} 5-\mathrm{C} 6-\mathrm{C} 7$ & $119.7(9)$ \\
\hline $\mathrm{C} 7-\mathrm{C} 6-\mathrm{H} 6$ & 120.1 \\
\hline $\mathrm{C} 6-\mathrm{C} 7-\mathrm{H} 7$ & 119.3 \\
\hline $\mathrm{C} 8-\mathrm{C} 7-\mathrm{C} 6$ & $121.4(8)$ \\
\hline & \\
\hline
\end{tabular}

\begin{tabular}{|c|c|}
\hline $\mathrm{C} 46-\mathrm{C} 45-\mathrm{H} 45 \mathrm{~A}$ & 109.4 \\
\hline $\mathrm{C} 46-\mathrm{C} 45-\mathrm{H} 45 \mathrm{~B}$ & 109.4 \\
\hline $\mathrm{C} 45-\mathrm{C} 46-\mathrm{H} 46 \mathrm{~A}$ & 109.6 \\
\hline $\mathrm{C} 45-\mathrm{C} 46-\mathrm{H} 46 \mathrm{~B}$ & 109.6 \\
\hline $\mathrm{C} 45-\mathrm{C} 46-\mathrm{C} 47$ & $110.2(9)$ \\
\hline $\mathrm{H} 46 \mathrm{~A}-\mathrm{C} 46-\mathrm{H} 46 \mathrm{~B}$ & 108.1 \\
\hline $\mathrm{C} 47-\mathrm{C} 46-\mathrm{H} 46 \mathrm{~A}$ & 109.6 \\
\hline $\mathrm{C} 47-\mathrm{C} 46-\mathrm{H} 46 \mathrm{~B}$ & 109.6 \\
\hline $\mathrm{C} 46-\mathrm{C} 47-\mathrm{H} 47 \mathrm{~A}$ & 109.1 \\
\hline $\mathrm{C} 46-\mathrm{C} 47-\mathrm{H} 47 \mathrm{~B}$ & 109.1 \\
\hline $\mathrm{C} 46-\mathrm{C} 47-\mathrm{C} 48$ & $112.4(9)$ \\
\hline $\mathrm{H} 47 \mathrm{~A}-\mathrm{C} 47-\mathrm{H} 47 \mathrm{~B}$ & 107.9 \\
\hline $\mathrm{C} 48-\mathrm{C} 47-\mathrm{H} 47 \mathrm{~A}$ & 109.1 \\
\hline $\mathrm{C} 48-\mathrm{C} 47-\mathrm{H} 47 \mathrm{~B}$ & 109.1 \\
\hline $\mathrm{C} 43-\mathrm{C} 48-\mathrm{H} 48 \mathrm{~A}$ & 109.7 \\
\hline $\mathrm{C} 43-\mathrm{C} 48-\mathrm{H} 48 \mathrm{~B}$ & 109.7 \\
\hline $\mathrm{C} 47-\mathrm{C} 48-\mathrm{C} 43$ & $109.9(8)$ \\
\hline $\mathrm{C} 47-\mathrm{C} 48-\mathrm{H} 48 \mathrm{~A}$ & 109.7 \\
\hline $\mathrm{C} 47-\mathrm{C} 48-\mathrm{H} 48 \mathrm{~B}$ & 109.7 \\
\hline $\mathrm{H} 48 \mathrm{~A}-\mathrm{C} 48-\mathrm{H} 48 \mathrm{~B}$ & 108.2 \\
\hline $\mathrm{P} 2-\mathrm{C} 49-\mathrm{H} 49$ & 105.9 \\
\hline $\mathrm{C} 50-\mathrm{C} 49-\mathrm{P} 2$ & $118.2(7)$ \\
\hline $\mathrm{C} 50-\mathrm{C} 49-\mathrm{H} 49$ & 105.9 \\
\hline $\mathrm{C} 54-\mathrm{C} 49-\mathrm{P} 2$ & $109.6(6)$ \\
\hline $\mathrm{C} 54-\mathrm{C} 49-\mathrm{H} 49$ & 105.9 \\
\hline $\mathrm{C} 54-\mathrm{C} 49-\mathrm{C} 50$ & $110.5(8)$ \\
\hline $\mathrm{C} 49-\mathrm{C} 50-\mathrm{H} 50 \mathrm{~A}$ & 109.7 \\
\hline $\mathrm{C} 49-\mathrm{C} 50-\mathrm{H} 50 \mathrm{~B}$ & 109.7 \\
\hline $\mathrm{H} 50 \mathrm{~A}-\mathrm{C} 50-\mathrm{H} 50 \mathrm{~B}$ & 108.2 \\
\hline $\mathrm{C} 51-\mathrm{C} 50-\mathrm{C} 49$ & $109.7(8)$ \\
\hline $\mathrm{C} 51-\mathrm{C} 50-\mathrm{H} 50 \mathrm{~A}$ & 109.7 \\
\hline $\mathrm{C} 51-\mathrm{C} 50-\mathrm{H} 50 \mathrm{~B}$ & 109.7 \\
\hline $\mathrm{C} 50-\mathrm{C} 51-\mathrm{H} 51 \mathrm{~A}$ & 109.4 \\
\hline $\mathrm{C} 50-\mathrm{C} 51-\mathrm{H} 51 \mathrm{~B}$ & 109.4 \\
\hline $\mathrm{H} 51 \mathrm{~A}-\mathrm{C} 51-\mathrm{H} 51 \mathrm{~B}$ & 108.0 \\
\hline $\mathrm{C} 52-\mathrm{C} 51-\mathrm{C} 50$ & $111.4(9)$ \\
\hline $\mathrm{C} 52-\mathrm{C} 51-\mathrm{H} 51 \mathrm{~A}$ & 109.4 \\
\hline $\mathrm{C} 52-\mathrm{C} 51-\mathrm{H} 51 \mathrm{~B}$ & 109.4 \\
\hline $\mathrm{C} 51-\mathrm{C} 52-\mathrm{H} 52 \mathrm{~A}$ & 109.4 \\
\hline C51-C52-H52B & 109.4 \\
\hline $\mathrm{C} 51-\mathrm{C} 52-\mathrm{C} 53$ & $111.0(8)$ \\
\hline $\mathrm{H} 52 \mathrm{~A}-\mathrm{C} 52-\mathrm{H} 52 \mathrm{~B}$ & 108.0 \\
\hline $\mathrm{C} 53-\mathrm{C} 52-\mathrm{H} 52 \mathrm{~A}$ & 109.4 \\
\hline $\mathrm{C} 53-\mathrm{C} 52-\mathrm{H} 52 \mathrm{~B}$ & 109.4 \\
\hline $\mathrm{C} 52-\mathrm{C} 53-\mathrm{H} 53 \mathrm{~A}$ & 109.2 \\
\hline $\mathrm{C} 52-\mathrm{C} 53-\mathrm{H} 53 \mathrm{~B}$ & 109.2 \\
\hline $\mathrm{C} 52-\mathrm{C} 53-\mathrm{C} 54$ & $112.2(9)$ \\
\hline $\mathrm{H} 53 \mathrm{~A}-\mathrm{C} 53-\mathrm{H} 53 \mathrm{~B}$ & 107.9 \\
\hline
\end{tabular}




\begin{tabular}{|c|c|}
\hline $\mathrm{C} 3-\mathrm{C} 8-\mathrm{C} 9$ & $124.3(8)$ \\
\hline $\mathrm{C} 7-\mathrm{C} 8-\mathrm{C} 3$ & $118.5(8)$ \\
\hline $\mathrm{C} 7-\mathrm{C} 8-\mathrm{C} 9$ & $117.2(7)$ \\
\hline $\mathrm{C} 10-\mathrm{C} 9-\mathrm{C} 8$ & $121.7(8)$ \\
\hline $\mathrm{C} 14-\mathrm{C} 9-\mathrm{C} 8$ & $119.9(8)$ \\
\hline $\mathrm{C} 14-\mathrm{C} 9-\mathrm{C} 10$ & $118.2(8)$ \\
\hline $\mathrm{O} 2-\mathrm{C} 10-\mathrm{C} 9$ & $114.5(7)$ \\
\hline $\mathrm{O} 2-\mathrm{C} 10-\mathrm{C} 11$ & $124.8(8)$ \\
\hline $\mathrm{C} 11-\mathrm{C} 10-\mathrm{C} 9$ & $120.7(8)$ \\
\hline $\mathrm{C} 10-\mathrm{C} 11-\mathrm{H} 11$ & 120.6 \\
\hline $\mathrm{C} 12-\mathrm{C} 11-\mathrm{C} 10$ & $118.9(9)$ \\
\hline $\mathrm{C} 12-\mathrm{C} 11-\mathrm{H} 11$ & 120.6 \\
\hline $\mathrm{C} 11-\mathrm{C} 12-\mathrm{H} 12$ & 119.0 \\
\hline $\mathrm{C} 13-\mathrm{C} 12-\mathrm{C} 11$ & $122.1(9)$ \\
\hline $\mathrm{C} 13-\mathrm{C} 12-\mathrm{H} 12$ & 119.0 \\
\hline $\mathrm{C} 12-\mathrm{C} 13-\mathrm{H} 13$ & 120.5 \\
\hline $\mathrm{C} 12-\mathrm{C} 13-\mathrm{C} 14$ & $119.0(9)$ \\
\hline $\mathrm{C} 14-\mathrm{C} 13-\mathrm{H} 13$ & 120.5 \\
\hline $\mathrm{O} 3-\mathrm{C} 14-\mathrm{C} 9$ & $115.0(8)$ \\
\hline $\mathrm{O} 3-\mathrm{C} 14-\mathrm{C} 13$ & $123.9(8)$ \\
\hline $\mathrm{C} 9-\mathrm{C} 14-\mathrm{C} 13$ & $121.1(8)$ \\
\hline $\mathrm{O} 2-\mathrm{C} 15-\mathrm{H} 15 \mathrm{~A}$ & 109.5 \\
\hline $\mathrm{O} 2-\mathrm{C} 15-\mathrm{H} 15 \mathrm{~B}$ & 109.5 \\
\hline $\mathrm{O} 2-\mathrm{C} 15-\mathrm{H} 15 \mathrm{C}$ & 109.5 \\
\hline $\mathrm{H} 15 \mathrm{~A}-\mathrm{C} 15-\mathrm{H} 15 \mathrm{~B}$ & 109.5 \\
\hline $\mathrm{H} 15 \mathrm{~A}-\mathrm{C} 15-\mathrm{H} 15 \mathrm{C}$ & 109.5 \\
\hline $\mathrm{H} 15 \mathrm{~B}-\mathrm{C} 15-\mathrm{H} 15 \mathrm{C}$ & 109.5 \\
\hline $\mathrm{O} 3-\mathrm{C} 16-\mathrm{H} 16 \mathrm{~A}$ & 109.5 \\
\hline $\mathrm{O} 3-\mathrm{C} 16-\mathrm{H} 16 \mathrm{~B}$ & 109.5 \\
\hline $\mathrm{O} 3-\mathrm{C} 16-\mathrm{H} 16 \mathrm{C}$ & 109.5 \\
\hline $\mathrm{H} 16 \mathrm{~A}-\mathrm{C} 16-\mathrm{H} 16 \mathrm{~B}$ & 109.5 \\
\hline $\mathrm{H} 16 \mathrm{~A}-\mathrm{C} 16-\mathrm{H} 16 \mathrm{C}$ & 109.5 \\
\hline $\mathrm{H} 16 \mathrm{~B}-\mathrm{C} 16-\mathrm{H} 16 \mathrm{C}$ & 109.5 \\
\hline $\mathrm{P} 1-\mathrm{C} 17-\mathrm{H} 17$ & 106.6 \\
\hline $\mathrm{C} 18-\mathrm{C} 17-\mathrm{P} 1$ & $116.5(6)$ \\
\hline $\mathrm{C} 18-\mathrm{C} 17-\mathrm{H} 17$ & 106.6 \\
\hline $\mathrm{C} 22-\mathrm{C} 17-\mathrm{P} 1$ & $109.7(6)$ \\
\hline $\mathrm{C} 22-\mathrm{C} 17-\mathrm{H} 17$ & 106.6 \\
\hline $\mathrm{C} 22-\mathrm{C} 17-\mathrm{C} 18$ & $110.1(7)$ \\
\hline $\mathrm{C} 17-\mathrm{C} 18-\mathrm{H} 18 \mathrm{~A}$ & 109.5 \\
\hline $\mathrm{C} 17-\mathrm{C} 18-\mathrm{H} 18 \mathrm{~B}$ & 109.5 \\
\hline $\mathrm{H} 18 \mathrm{~A}-\mathrm{C} 18-\mathrm{H} 18 \mathrm{~B}$ & 108.1 \\
\hline $\mathrm{C} 19-\mathrm{C} 18-\mathrm{C} 17$ & $110.8(8)$ \\
\hline $\mathrm{C} 19-\mathrm{C} 18-\mathrm{H} 18 \mathrm{~A}$ & 109.5 \\
\hline $\mathrm{C} 19-\mathrm{C} 18-\mathrm{H} 18 \mathrm{~B}$ & 109.5 \\
\hline $\mathrm{C} 18-\mathrm{C} 19-\mathrm{H} 19 \mathrm{~A}$ & 109.3 \\
\hline $\mathrm{C} 18-\mathrm{C} 19-\mathrm{H} 19 \mathrm{~B}$ & 109.3 \\
\hline & \\
\hline
\end{tabular}

\begin{tabular}{|c|c|}
\hline $\mathrm{C} 54-\mathrm{C} 53-\mathrm{H} 53 \mathrm{~A}$ & 109.2 \\
\hline $\mathrm{C} 54-\mathrm{C} 53-\mathrm{H} 53 \mathrm{~B}$ & 109.2 \\
\hline $\mathrm{C} 49-\mathrm{C} 54-\mathrm{C} 53$ & $110.1(8)$ \\
\hline $\mathrm{C} 49-\mathrm{C} 54-\mathrm{H} 54 \mathrm{~A}$ & 109.6 \\
\hline $\mathrm{C} 49-\mathrm{C} 54-\mathrm{H} 54 \mathrm{~B}$ & 109.6 \\
\hline $\mathrm{C} 53-\mathrm{C} 54-\mathrm{H} 54 \mathrm{~A}$ & 109.6 \\
\hline C53-C $54-\mathrm{H} 54 \mathrm{~B}$ & 109.6 \\
\hline $\mathrm{H} 54 \mathrm{~A}-\mathrm{C} 54-\mathrm{H} 54 \mathrm{~B}$ & 108.2 \\
\hline $\mathrm{C} 56-\mathrm{C} 55-\mathrm{P} 3$ & $118.8(7)$ \\
\hline $\mathrm{C} 56-\mathrm{C} 55-\mathrm{C} 60$ & $118.6(8)$ \\
\hline $\mathrm{C} 60-\mathrm{C} 55-\mathrm{P} 3$ & $122.5(7)$ \\
\hline $\mathrm{C} 55-\mathrm{C} 56-\mathrm{H} 56$ & 119.0 \\
\hline $\mathrm{C} 57-\mathrm{C} 56-\mathrm{C} 55$ & $122.0(9)$ \\
\hline $\mathrm{C} 57-\mathrm{C} 56-\mathrm{H} 56$ & 119.0 \\
\hline $\mathrm{C} 56-\mathrm{C} 57-\mathrm{H} 57$ & 120.5 \\
\hline $\mathrm{C} 58-\mathrm{C} 57-\mathrm{C} 56$ & $119.0(9)$ \\
\hline $\mathrm{C} 58-\mathrm{C} 57-\mathrm{H} 57$ & 120.5 \\
\hline $\mathrm{C} 57-\mathrm{C} 58-\mathrm{H} 58$ & 119.9 \\
\hline $\mathrm{C} 57-\mathrm{C} 58-\mathrm{C} 59$ & $120.3(9)$ \\
\hline $\mathrm{C} 59-\mathrm{C} 58-\mathrm{H} 58$ & 119.9 \\
\hline $\mathrm{C} 58-\mathrm{C} 59-\mathrm{H} 59$ & 119.1 \\
\hline $\mathrm{C} 60-\mathrm{C} 59-\mathrm{C} 58$ & $121.7(9)$ \\
\hline $\mathrm{C} 60-\mathrm{C} 59-\mathrm{H} 59$ & 119.1 \\
\hline $\mathrm{C} 55-\mathrm{C} 60-\mathrm{C} 61$ & $124.5(8)$ \\
\hline $\mathrm{C} 59-\mathrm{C} 60-\mathrm{C} 55$ & $118.3(9)$ \\
\hline $\mathrm{C} 59-\mathrm{C} 60-\mathrm{C} 61$ & $117.0(8)$ \\
\hline $\mathrm{C} 62-\mathrm{C} 61-\mathrm{C} 60$ & $122.8(8)$ \\
\hline $\mathrm{C} 62-\mathrm{C} 61-\mathrm{C} 66$ & $118.0(9)$ \\
\hline $\mathrm{C} 66-\mathrm{C} 61-\mathrm{C} 60$ & $119.0(8)$ \\
\hline $\mathrm{O} 6-\mathrm{C} 62-\mathrm{C} 61$ & $115.3(8)$ \\
\hline $\mathrm{O} 6-\mathrm{C} 62-\mathrm{C} 63$ & $123.3(8)$ \\
\hline $\mathrm{C} 63-\mathrm{C} 62-\mathrm{C} 61$ & $121.4(9)$ \\
\hline C62-C63-H63 & 120.8 \\
\hline $\mathrm{C} 64-\mathrm{C} 63-\mathrm{C} 62$ & $118.4(10)$ \\
\hline $\mathrm{C} 64-\mathrm{C} 63-\mathrm{H} 63$ & 120.8 \\
\hline $\mathrm{C} 63-\mathrm{C} 64-\mathrm{H} 64$ & 118.9 \\
\hline $\mathrm{C} 65-\mathrm{C} 64-\mathrm{C} 63$ & $122.2(9)$ \\
\hline $\mathrm{C} 65-\mathrm{C} 64-\mathrm{H} 64$ & 118.9 \\
\hline $\mathrm{C} 64-\mathrm{C} 65-\mathrm{H} 65$ & 120.5 \\
\hline $\mathrm{C} 64-\mathrm{C} 65-\mathrm{C} 66$ & $119.0(9)$ \\
\hline $\mathrm{C} 66-\mathrm{C} 65-\mathrm{H} 65$ & 120.5 \\
\hline $\mathrm{O} 7-\mathrm{C} 66-\mathrm{C} 61$ & $114.4(8)$ \\
\hline $\mathrm{C} 65-\mathrm{C} 66-\mathrm{O} 7$ & $124.5(9)$ \\
\hline $\mathrm{C} 65-\mathrm{C} 66-\mathrm{C} 61$ & $121.0(9)$ \\
\hline $\mathrm{O} 6-\mathrm{C} 67-\mathrm{H} 67 \mathrm{~A}$ & 109.5 \\
\hline $\mathrm{O} 6-\mathrm{C} 67-\mathrm{H} 67 \mathrm{~B}$ & 109.5 \\
\hline $\mathrm{O} 6-\mathrm{C} 67-\mathrm{H} 67 \mathrm{C}$ & 109.5 \\
\hline $\mathrm{H} 67 \mathrm{~A}-\mathrm{C} 67-\mathrm{H} 67 \mathrm{~B}$ & 109.5 \\
\hline
\end{tabular}




\begin{tabular}{|c|c|}
\hline $\mathrm{C} 20-\mathrm{C} 19-\mathrm{C} 18$ & $111.7(8)$ \\
\hline $\mathrm{C} 20-\mathrm{C} 19-\mathrm{H} 19 \mathrm{~A}$ & 109.3 \\
\hline $\mathrm{C} 20-\mathrm{C} 19-\mathrm{H} 19 \mathrm{~B}$ & 109.3 \\
\hline $\mathrm{C} 19-\mathrm{C} 20-\mathrm{H} 20 \mathrm{~A}$ & 109.1 \\
\hline $\mathrm{C} 19-\mathrm{C} 20-\mathrm{H} 20 \mathrm{~B}$ & 109.1 \\
\hline $\mathrm{H} 20 \mathrm{~A}-\mathrm{C} 20-\mathrm{H} 20 \mathrm{~B}$ & 107.9 \\
\hline $\mathrm{C} 21-\mathrm{C} 20-\mathrm{C} 19$ & $112.3(9)$ \\
\hline $\mathrm{C} 21-\mathrm{C} 20-\mathrm{H} 20 \mathrm{~A}$ & 109.1 \\
\hline $\mathrm{C} 21-\mathrm{C} 20-\mathrm{H} 20 \mathrm{~B}$ & 109.1 \\
\hline $\mathrm{C} 20-\mathrm{C} 21-\mathrm{H} 21 \mathrm{~A}$ & 109.6 \\
\hline $\mathrm{C} 20-\mathrm{C} 21-\mathrm{H} 21 \mathrm{~B}$ & 109.6 \\
\hline $\mathrm{C} 20-\mathrm{C} 21-\mathrm{C} 22$ & $110.5(8)$ \\
\hline $\mathrm{H} 21 \mathrm{~A}-\mathrm{C} 21-\mathrm{H} 21 \mathrm{~B}$ & 108.1 \\
\hline $\mathrm{C} 22-\mathrm{C} 21-\mathrm{H} 21 \mathrm{~A}$ & 109.6 \\
\hline $\mathrm{C} 22-\mathrm{C} 21-\mathrm{H} 21 \mathrm{~B}$ & 109.6 \\
\hline $\mathrm{C} 17-\mathrm{C} 22-\mathrm{C} 21$ & $112.5(8)$ \\
\hline $\mathrm{C} 17-\mathrm{C} 22-\mathrm{H} 22 \mathrm{~A}$ & 109.1 \\
\hline $\mathrm{C} 17-\mathrm{C} 22-\mathrm{H} 22 \mathrm{~B}$ & 109.1 \\
\hline $\mathrm{C} 21-\mathrm{C} 22-\mathrm{H} 22 \mathrm{~A}$ & 109.1 \\
\hline $\mathrm{C} 21-\mathrm{C} 22-\mathrm{H} 22 \mathrm{~B}$ & 109.1 \\
\hline $\mathrm{H} 22 \mathrm{~A}-\mathrm{C} 22-\mathrm{H} 22 \mathrm{~B}$ & 107.8 \\
\hline $\mathrm{P} 1-\mathrm{C} 23-\mathrm{H} 23$ & 108.3 \\
\hline $\mathrm{C} 24-\mathrm{C} 23-\mathrm{P} 1$ & $110.5(6)$ \\
\hline $\mathrm{C} 24-\mathrm{C} 23-\mathrm{H} 23$ & 108.3 \\
\hline $\mathrm{C} 28-\mathrm{C} 23-\mathrm{P} 1$ & $112.5(5)$ \\
\hline $\mathrm{C} 28-\mathrm{C} 23-\mathrm{H} 23$ & 108.3 \\
\hline $\mathrm{C} 28-\mathrm{C} 23-\mathrm{C} 24$ & $108.8(7)$ \\
\hline $\mathrm{C} 23-\mathrm{C} 24-\mathrm{H} 24 \mathrm{~A}$ & 109.6 \\
\hline $\mathrm{C} 23-\mathrm{C} 24-\mathrm{H} 24 \mathrm{~B}$ & 109.6 \\
\hline $\mathrm{H} 24 \mathrm{~A}-\mathrm{C} 24-\mathrm{H} 24 \mathrm{~B}$ & 108.1 \\
\hline $\mathrm{C} 25-\mathrm{C} 24-\mathrm{C} 23$ & $110.1(7)$ \\
\hline $\mathrm{C} 25-\mathrm{C} 24-\mathrm{H} 24 \mathrm{~A}$ & 109.6 \\
\hline $\mathrm{C} 25-\mathrm{C} 24-\mathrm{H} 24 \mathrm{~B}$ & 109.6 \\
\hline $\mathrm{C} 24-\mathrm{C} 25-\mathrm{H} 25 \mathrm{~A}$ & 109.4 \\
\hline $\mathrm{C} 24-\mathrm{C} 25-\mathrm{H} 25 \mathrm{~B}$ & 109.4 \\
\hline $\mathrm{H} 25 \mathrm{~A}-\mathrm{C} 25-\mathrm{H} 25 \mathrm{~B}$ & 108.0 \\
\hline $\mathrm{C} 26-\mathrm{C} 25-\mathrm{C} 24$ & $111.3(7)$ \\
\hline $\mathrm{C} 26-\mathrm{C} 25-\mathrm{H} 25 \mathrm{~A}$ & 109.4 \\
\hline $\mathrm{C} 26-\mathrm{C} 25-\mathrm{H} 25 \mathrm{~B}$ & 109.4 \\
\hline $\mathrm{C} 25-\mathrm{C} 26-\mathrm{H} 26 \mathrm{~A}$ & 109.3 \\
\hline $\mathrm{C} 25-\mathrm{C} 26-\mathrm{H} 26 \mathrm{~B}$ & 109.3 \\
\hline $\mathrm{H} 26 \mathrm{~A}-\mathrm{C} 26-\mathrm{H} 26 \mathrm{~B}$ & 108.0 \\
\hline $\mathrm{C} 27-\mathrm{C} 26-\mathrm{C} 25$ & $111.4(8)$ \\
\hline $\mathrm{C} 27-\mathrm{C} 26-\mathrm{H} 26 \mathrm{~A}$ & 109.3 \\
\hline $\mathrm{C} 27-\mathrm{C} 26-\mathrm{H} 26 \mathrm{~B}$ & 109.3 \\
\hline $\mathrm{C} 26-\mathrm{C} 27-\mathrm{H} 27 \mathrm{~A}$ & 109.3 \\
\hline $\mathrm{C} 26-\mathrm{C} 27-\mathrm{H} 27 \mathrm{~B}$ & 109.3 \\
\hline & \\
\hline
\end{tabular}

\begin{tabular}{|c|c|}
\hline $\mathrm{H} 67 \mathrm{~A}-\mathrm{C} 67-\mathrm{H} 67 \mathrm{C}$ & 109.5 \\
\hline $\mathrm{H} 67 \mathrm{~B}-\mathrm{C} 67-\mathrm{H} 67 \mathrm{C}$ & 109.5 \\
\hline $\mathrm{O} 7-\mathrm{C} 68-\mathrm{H} 68 \mathrm{~A}$ & 109.5 \\
\hline $\mathrm{O} 7-\mathrm{C} 68-\mathrm{H} 68 \mathrm{~B}$ & 109.5 \\
\hline $\mathrm{O} 7-\mathrm{C} 68-\mathrm{H} 68 \mathrm{C}$ & 109.5 \\
\hline $\mathrm{H} 68 \mathrm{~A}-\mathrm{C} 68-\mathrm{H} 68 \mathrm{~B}$ & 109.5 \\
\hline $\mathrm{H} 68 \mathrm{~A}-\mathrm{C} 68-\mathrm{H} 68 \mathrm{C}$ & 109.5 \\
\hline $\mathrm{H} 68 \mathrm{~B}-\mathrm{C} 68-\mathrm{H} 68 \mathrm{C}$ & 109.5 \\
\hline P3-C69-H69 & 108.0 \\
\hline $\mathrm{C} 70-\mathrm{C} 69-\mathrm{P} 3$ & $111.2(7)$ \\
\hline $\mathrm{C} 70-\mathrm{C} 69-\mathrm{H} 69$ & 108.0 \\
\hline $\mathrm{C} 70-\mathrm{C} 69-\mathrm{C} 74$ & $109.6(8)$ \\
\hline $\mathrm{C} 74-\mathrm{C} 69-\mathrm{P} 3$ & $111.8(6)$ \\
\hline C74-C69-H69 & 108.0 \\
\hline $\mathrm{C} 69-\mathrm{C} 70-\mathrm{H} 70 \mathrm{~A}$ & 109.8 \\
\hline $\mathrm{C} 69-\mathrm{C} 70-\mathrm{H} 70 \mathrm{~B}$ & 109.8 \\
\hline $\mathrm{C} 69-\mathrm{C} 70-\mathrm{C} 71$ & $109.6(9)$ \\
\hline $\mathrm{H} 70 \mathrm{~A}-\mathrm{C} 70-\mathrm{H} 70 \mathrm{~B}$ & 108.2 \\
\hline $\mathrm{C} 71-\mathrm{C} 70-\mathrm{H} 70 \mathrm{~A}$ & 109.8 \\
\hline $\mathrm{C} 71-\mathrm{C} 70-\mathrm{H} 70 \mathrm{~B}$ & 109.8 \\
\hline $\mathrm{C} 70-\mathrm{C} 71-\mathrm{H} 71 \mathrm{~A}$ & 109.2 \\
\hline $\mathrm{C} 70-\mathrm{C} 71-\mathrm{H} 71 \mathrm{~B}$ & 109.2 \\
\hline $\mathrm{H} 71 \mathrm{~A}-\mathrm{C} 71-\mathrm{H} 71 \mathrm{~B}$ & 107.9 \\
\hline $\mathrm{C} 72-\mathrm{C} 71-\mathrm{C} 70$ & $112.2(9)$ \\
\hline $\mathrm{C} 72-\mathrm{C} 71-\mathrm{H} 71 \mathrm{~A}$ & 109.2 \\
\hline $\mathrm{C} 72-\mathrm{C} 71-\mathrm{H} 71 \mathrm{~B}$ & 109.2 \\
\hline $\mathrm{C} 71-\mathrm{C} 72-\mathrm{H} 72 \mathrm{~A}$ & 109.0 \\
\hline $\mathrm{C} 71-\mathrm{C} 72-\mathrm{H} 72 \mathrm{~B}$ & 109.0 \\
\hline $\mathrm{H} 72 \mathrm{~A}-\mathrm{C} 72-\mathrm{H} 72 \mathrm{~B}$ & 107.8 \\
\hline $\mathrm{C} 73-\mathrm{C} 72-\mathrm{C} 71$ & $113.1(10)$ \\
\hline $\mathrm{C} 73-\mathrm{C} 72-\mathrm{H} 72 \mathrm{~A}$ & 109.0 \\
\hline C73-C72-H72B & 109.0 \\
\hline $\mathrm{C} 72-\mathrm{C} 73-\mathrm{H} 73 \mathrm{~A}$ & 109.3 \\
\hline $\mathrm{C} 72-\mathrm{C} 73-\mathrm{H} 73 \mathrm{~B}$ & 109.3 \\
\hline $\mathrm{C} 72-\mathrm{C} 73-\mathrm{C} 74$ & $111.5(9)$ \\
\hline $\mathrm{H} 73 \mathrm{~A}-\mathrm{C} 73-\mathrm{H} 73 \mathrm{~B}$ & 108.0 \\
\hline $\mathrm{C} 74-\mathrm{C} 73-\mathrm{H} 73 \mathrm{~A}$ & 109.3 \\
\hline $\mathrm{C} 74-\mathrm{C} 73-\mathrm{H} 73 \mathrm{~B}$ & 109.3 \\
\hline $\mathrm{C} 69-\mathrm{C} 74-\mathrm{H} 74 \mathrm{~A}$ & 109.5 \\
\hline $\mathrm{C} 69-\mathrm{C} 74-\mathrm{H} 74 \mathrm{~B}$ & 109.5 \\
\hline $\mathrm{C} 73-\mathrm{C} 74-\mathrm{C} 69$ & $110.8(9)$ \\
\hline $\mathrm{C} 73-\mathrm{C} 74-\mathrm{H} 74 \mathrm{~A}$ & 109.5 \\
\hline $\mathrm{C} 73-\mathrm{C} 74-\mathrm{H} 74 \mathrm{~B}$ & 109.5 \\
\hline $\mathrm{H} 74 \mathrm{~A}-\mathrm{C} 74-\mathrm{H} 74 \mathrm{~B}$ & 108.1 \\
\hline $\mathrm{P} 3-\mathrm{C} 75-\mathrm{H} 75$ & 104.2 \\
\hline $\mathrm{C} 76-\mathrm{C} 75-\mathrm{P} 3$ & $115.7(8)$ \\
\hline $\mathrm{C} 76-\mathrm{C} 75-\mathrm{H} 75$ & 104.2 \\
\hline $\mathrm{C} 80-\mathrm{C} 75-\mathrm{P} 3$ & $114.2(9)$ \\
\hline
\end{tabular}




\begin{tabular}{|c|c|}
\hline $\mathrm{C} 28-\mathrm{C} 27-\mathrm{C} 26$ & $111.7(7)$ \\
\hline $\mathrm{C} 28-\mathrm{C} 27-\mathrm{H} 27 \mathrm{~A}$ & 109.3 \\
\hline $\mathrm{C} 28-\mathrm{C} 27-\mathrm{H} 27 \mathrm{~B}$ & 109.3 \\
\hline $\mathrm{C} 23-\mathrm{C} 28-\mathrm{H} 28 \mathrm{~A}$ & 109.5 \\
\hline $\mathrm{C} 23-\mathrm{C} 28-\mathrm{H} 28 \mathrm{~B}$ & 109.5 \\
\hline $\mathrm{C} 27-\mathrm{C} 28-\mathrm{C} 23$ & $110.7(7)$ \\
\hline $\mathrm{C} 27-\mathrm{C} 28-\mathrm{H} 28 \mathrm{~A}$ & 109.5 \\
\hline $\mathrm{C} 27-\mathrm{C} 28-\mathrm{H} 28 \mathrm{~B}$ & 109.5 \\
\hline $\mathrm{H} 28 \mathrm{~A}-\mathrm{C} 28-\mathrm{H} 28 \mathrm{~B}$ & 108.1 \\
\hline $\mathrm{C} 30-\mathrm{C} 29-\mathrm{P} 2$ & $117.2(7)$ \\
\hline $\mathrm{C} 34-\mathrm{C} 29-\mathrm{P} 2$ & $124.2(7)$ \\
\hline $\mathrm{C} 34-\mathrm{C} 29-\mathrm{C} 30$ & $118.4(9)$ \\
\hline $\mathrm{C} 29-\mathrm{C} 30-\mathrm{H} 30$ & 119.5 \\
\hline $\mathrm{C} 31-\mathrm{C} 30-\mathrm{C} 29$ & $121.1(10)$ \\
\hline $\mathrm{C} 31-\mathrm{C} 30-\mathrm{H} 30$ & 119.5 \\
\hline $\mathrm{C} 30-\mathrm{C} 31-\mathrm{H} 31$ & 120.4 \\
\hline $\mathrm{C} 30-\mathrm{C} 31-\mathrm{C} 32$ & $119.3(11)$ \\
\hline $\mathrm{C} 32-\mathrm{C} 31-\mathrm{H} 31$ & 120.4 \\
\hline $\mathrm{C} 31-\mathrm{C} 32-\mathrm{H} 32$ & 120.2 \\
\hline $\mathrm{C} 33-\mathrm{C} 32-\mathrm{C} 31$ & $119.7(10)$ \\
\hline $\mathrm{C} 33-\mathrm{C} 32-\mathrm{H} 32$ & 120.2 \\
\hline $\mathrm{C} 32-\mathrm{C} 33-\mathrm{H} 33$ & 118.9 \\
\hline $\mathrm{C} 32-\mathrm{C} 33-\mathrm{C} 34$ & $122.1(10)$ \\
\hline $\mathrm{C} 34-\mathrm{C} 33-\mathrm{H} 33$ & 118.9 \\
\hline $\mathrm{C} 29-\mathrm{C} 34-\mathrm{C} 35$ & $122.3(9)$ \\
\hline $\mathrm{C} 33-\mathrm{C} 34-\mathrm{C} 29$ & $119.2(10)$ \\
\hline $\mathrm{C} 33-\mathrm{C} 34-\mathrm{C} 35$ & $118.5(9)$ \\
\hline $\mathrm{C} 36-\mathrm{C} 35-\mathrm{C} 34$ & $119.7(11)$ \\
\hline $\mathrm{C} 36-\mathrm{C} 35-\mathrm{C} 40$ & $117.7(11)$ \\
\hline $\mathrm{C} 40-\mathrm{C} 35-\mathrm{C} 34$ & $122.3(10)$ \\
\hline $\mathrm{C} 35-\mathrm{C} 36-\mathrm{O} 4$ & $117.6(12)$ \\
\hline $\mathrm{C} 35-\mathrm{C} 36-\mathrm{C} 37$ & $123.3(14)$ \\
\hline $\mathrm{C} 37-\mathrm{C} 36-\mathrm{O} 4$ & $119.0(13)$ \\
\hline $\mathrm{C} 36-\mathrm{C} 37-\mathrm{H} 37$ & 121.7 \\
\hline $\mathrm{C} 36-\mathrm{C} 37-\mathrm{C} 38$ & $116.7(14)$ \\
\hline $\mathrm{C} 38-\mathrm{C} 37-\mathrm{H} 37$ & 121.7 \\
\hline $\mathrm{C} 37-\mathrm{C} 38-\mathrm{H} 38$ & 119.0 \\
\hline $\mathrm{C} 39-\mathrm{C} 38-\mathrm{C} 37$ & $122.0(13)$ \\
\hline $\mathrm{C} 39-\mathrm{C} 38-\mathrm{H} 38$ & 119.0 \\
\hline $\mathrm{C} 38-\mathrm{C} 39-\mathrm{H} 39$ & 120.6 \\
\hline $\mathrm{C} 38-\mathrm{C} 39-\mathrm{C} 40$ & $118.7(13)$ \\
\hline $\mathrm{C} 40-\mathrm{C} 39-\mathrm{H} 39$ & 120.6 \\
\hline $\mathrm{O} 5-\mathrm{C} 40-\mathrm{C} 35$ & $114.2(10)$ \\
\hline $\mathrm{O} 5-\mathrm{C} 40-\mathrm{C} 39$ & $124.3(12)$ \\
\hline $\mathrm{C} 35-\mathrm{C} 40-\mathrm{C} 39$ & $121.4(12)$ \\
\hline $\mathrm{O} 4-\mathrm{C} 41-\mathrm{H} 41 \mathrm{~A}$ & 109.5 \\
\hline $\mathrm{O} 4-\mathrm{C} 41-\mathrm{H} 41 \mathrm{~B}$ & 109.5 \\
\hline $\mathrm{O} 4-\mathrm{C}$ & \\
\hline
\end{tabular}

\begin{tabular}{|c|c|}
\hline $\mathrm{C} 80-\mathrm{C} 75-\mathrm{H} 75$ & 104.2 \\
\hline $\mathrm{C} 80-\mathrm{C} 75-\mathrm{C} 76$ & $112.5(11)$ \\
\hline $\mathrm{C} 75-\mathrm{C} 76-\mathrm{H} 76 \mathrm{~A}$ & 108.6 \\
\hline $\mathrm{C} 75-\mathrm{C} 76-\mathrm{H} 76 \mathrm{~B}$ & 108.6 \\
\hline $\mathrm{C} 75-\mathrm{C} 76-\mathrm{C} 77$ & $114.8(11)$ \\
\hline $\mathrm{H} 76 \mathrm{~A}-\mathrm{C} 76-\mathrm{H} 76 \mathrm{~B}$ & 107.5 \\
\hline $\mathrm{C} 77-\mathrm{C} 76-\mathrm{H} 76 \mathrm{~A}$ & 108.6 \\
\hline $\mathrm{C} 77-\mathrm{C} 76-\mathrm{H} 76 \mathrm{~B}$ & 108.6 \\
\hline $\mathrm{C} 76-\mathrm{C} 77-\mathrm{H} 77 \mathrm{~A}$ & 109.2 \\
\hline $\mathrm{C} 76-\mathrm{C} 77-\mathrm{H} 77 \mathrm{~B}$ & 109.2 \\
\hline $\mathrm{H} 77 \mathrm{~A}-\mathrm{C} 77-\mathrm{H} 77 \mathrm{~B}$ & 107.9 \\
\hline $\mathrm{C} 78-\mathrm{C} 77-\mathrm{C} 76$ & $112.0(12)$ \\
\hline $\mathrm{C} 78-\mathrm{C} 77-\mathrm{H} 77 \mathrm{~A}$ & 109.2 \\
\hline $\mathrm{C} 78-\mathrm{C} 77-\mathrm{H} 77 \mathrm{~B}$ & 109.2 \\
\hline $\mathrm{C} 77-\mathrm{C} 78-\mathrm{H} 78 \mathrm{~A}$ & 108.3 \\
\hline $\mathrm{C} 77-\mathrm{C} 78-\mathrm{H} 78 \mathrm{~B}$ & 108.3 \\
\hline $\mathrm{C} 77-\mathrm{C} 78-\mathrm{C} 79$ & $115.9(13)$ \\
\hline $\mathrm{H} 78 \mathrm{~A}-\mathrm{C} 78-\mathrm{H} 78 \mathrm{~B}$ & 107.4 \\
\hline $\mathrm{C} 79-\mathrm{C} 78-\mathrm{H} 78 \mathrm{~A}$ & 108.3 \\
\hline $\mathrm{C} 79-\mathrm{C} 78-\mathrm{H} 78 \mathrm{~B}$ & 108.3 \\
\hline $\mathrm{C} 78-\mathrm{C} 79-\mathrm{H} 79 \mathrm{~A}$ & 110.2 \\
\hline $\mathrm{C} 78-\mathrm{C} 79-\mathrm{H} 79 \mathrm{~B}$ & 110.2 \\
\hline $\mathrm{H} 79 \mathrm{~A}-\mathrm{C} 79-\mathrm{H} 79 \mathrm{~B}$ & 108.5 \\
\hline $\mathrm{C} 80-\mathrm{C} 79-\mathrm{C} 78$ & $107.5(13)$ \\
\hline $\mathrm{C} 80-\mathrm{C} 79-\mathrm{H} 79 \mathrm{~A}$ & 110.2 \\
\hline $\mathrm{C} 80-\mathrm{C} 79-\mathrm{H} 79 \mathrm{~B}$ & 110.2 \\
\hline $\mathrm{C} 75-\mathrm{C} 80-\mathrm{C} 79$ & $114.5(12)$ \\
\hline $\mathrm{C} 75-\mathrm{C} 80-\mathrm{H} 80 \mathrm{~A}$ & 108.6 \\
\hline $\mathrm{C} 75-\mathrm{C} 80-\mathrm{H} 80 \mathrm{~B}$ & 108.6 \\
\hline $\mathrm{C} 79-\mathrm{C} 80-\mathrm{H} 80 \mathrm{~A}$ & 108.6 \\
\hline $\mathrm{C} 79-\mathrm{C} 80-\mathrm{H} 80 \mathrm{~B}$ & 108.6 \\
\hline $\mathrm{H} 80 \mathrm{~A}-\mathrm{C} 80-\mathrm{H} 80 \mathrm{~B}$ & 107.6 \\
\hline $\mathrm{O} 1 \mathrm{~A}-\mathrm{S} 1 \mathrm{~A}-\mathrm{N} 1 \mathrm{~A}$ & $116.1(6)$ \\
\hline $\mathrm{O} 1 \mathrm{~A}-\mathrm{S} 1 \mathrm{~A}-\mathrm{C} 1 \mathrm{~A}$ & $105.4(6)$ \\
\hline $\mathrm{O} 2 \mathrm{~A}-\mathrm{S} 1 \mathrm{~A}-\mathrm{O} 1 \mathrm{~A}$ & $121.1(6)$ \\
\hline $\mathrm{O} 2 \mathrm{~A}-\mathrm{S} 1 \mathrm{~A}-\mathrm{N} 1 \mathrm{~A}$ & $107.9(6)$ \\
\hline $\mathrm{O} 2 \mathrm{~A}-\mathrm{S} 1 \mathrm{~A}-\mathrm{C} 1 \mathrm{~A}$ & $101.7(6)$ \\
\hline $\mathrm{N} 1 \mathrm{~A}-\mathrm{S} 1 \mathrm{~A}-\mathrm{C} 1 \mathrm{~A}$ & $101.8(6)$ \\
\hline $\mathrm{O} 3 \mathrm{~A}-\mathrm{S} 2 \mathrm{~A}-\mathrm{O} 4 \mathrm{~A}$ & $121.5(6)$ \\
\hline $\mathrm{O} 3 \mathrm{~A}-\mathrm{S} 2 \mathrm{~A}-\mathrm{N} 1 \mathrm{~A}$ & $115.4(6)$ \\
\hline $\mathrm{O} 3 \mathrm{~A}-\mathrm{S} 2 \mathrm{~A}-\mathrm{C} 2 \mathrm{~A}$ & $102.3(6)$ \\
\hline $\mathrm{O} 4 \mathrm{~A}-\mathrm{S} 2 \mathrm{~A}-\mathrm{N} 1 \mathrm{~A}$ & $111.2(5)$ \\
\hline $\mathrm{O} 4 \mathrm{~A}-\mathrm{S} 2 \mathrm{~A}-\mathrm{C} 2 \mathrm{~A}$ & $100.6(6)$ \\
\hline $\mathrm{N} 1 \mathrm{~A}-\mathrm{S} 2 \mathrm{~A}-\mathrm{C} 2 \mathrm{~A}$ & $101.8(6)$ \\
\hline $\mathrm{S} 2 \mathrm{~A}-\mathrm{N} 1 \mathrm{~A}-\mathrm{S} 1 \mathrm{~A}$ & $126.9(7)$ \\
\hline $\mathrm{F} 1 \mathrm{~A}-\mathrm{C} 1 \mathrm{~A}-\mathrm{S} 1 \mathrm{~A}$ & $113.1(9)$ \\
\hline $\mathrm{F} 1 \mathrm{~A}-\mathrm{C} 1 \mathrm{~A}-\mathrm{F} 2 \mathrm{~A}$ & $108.9(11)$ \\
\hline $\mathrm{F} 1 \mathrm{~A}-\mathrm{C} 1 \mathrm{~A}-\mathrm{F} 3 \mathrm{~A}$ & 106.9 \\
\hline
\end{tabular}




\begin{tabular}{|c|c|c|c|}
\hline $\mathrm{H} 41 \mathrm{~A}-\mathrm{C} 41-\mathrm{H} 41 \mathrm{~B}$ & 109.5 & $\mathrm{~F} 2 \mathrm{~A}-\mathrm{C} 1 \mathrm{~A}-\mathrm{S} 1 \mathrm{~A}$ & $110.4(9)$ \\
\hline $\mathrm{H} 41 \mathrm{~A}-\mathrm{C} 41-\mathrm{H} 41 \mathrm{C}$ & 109.5 & $\mathrm{~F} 2 \mathrm{~A}-\mathrm{C} 1 \mathrm{~A}-\mathrm{F} 3 \mathrm{~A}$ & $104.9(10)$ \\
\hline $\mathrm{H} 41 \mathrm{~B}-\mathrm{C} 41-\mathrm{H} 41 \mathrm{C}$ & 109.5 & $\mathrm{~F} 3 \mathrm{~A}-\mathrm{C} 1 \mathrm{~A}-\mathrm{S} 1 \mathrm{~A}$ & $112.2(9)$ \\
\hline $\mathrm{O} 5-\mathrm{C} 42-\mathrm{H} 42 \mathrm{~A}$ & 109.5 & $\mathrm{~F} 4 \mathrm{~A}-\mathrm{C} 2 \mathrm{~A}-\mathrm{S} 2 \mathrm{~A}$ & $115.4(9)$ \\
\hline $\mathrm{O} 5-\mathrm{C} 42-\mathrm{H} 42 \mathrm{~B}$ & 109.5 & $\mathrm{~F} 4 \mathrm{~A}-\mathrm{C} 2 \mathrm{~A}-\mathrm{F} 5 \mathrm{~A}$ & $106.6(11)$ \\
\hline $\mathrm{O} 5-\mathrm{C} 42-\mathrm{H} 42 \mathrm{C}$ & 109.5 & $\mathrm{~F} 4 \mathrm{~A}-\mathrm{C} 2 \mathrm{~A}-\mathrm{F} 6 \mathrm{~A}$ & $108.2(12)$ \\
\hline $\mathrm{H} 42 \mathrm{~A}-\mathrm{C} 42-\mathrm{H} 42 \mathrm{~B}$ & 109.5 & $\mathrm{~F} 5 \mathrm{~A}-\mathrm{C} 2 \mathrm{~A}-\mathrm{S} 2 \mathrm{~A}$ & $109.1(9)$ \\
\hline $\mathrm{H} 42 \mathrm{~A}-\mathrm{C} 42-\mathrm{H} 42 \mathrm{C}$ & 109.5 & $\mathrm{~F} 5 \mathrm{~A}-\mathrm{C} 2 \mathrm{~A}-\mathrm{F} 6 \mathrm{~A}$ & $106.3(10)$ \\
\hline $\mathrm{H} 42 \mathrm{~B}-\mathrm{C} 42-\mathrm{H} 42 \mathrm{C}$ & 109.5 & $\mathrm{~F} 6 \mathrm{~A}-\mathrm{C} 2 \mathrm{~A}-\mathrm{S} 2 \mathrm{~A}$ & $110.8(9)$ \\
\hline $\mathrm{Au} 1-\mathrm{P} 1-\mathrm{C} 3-\mathrm{C} 4$ & $161.8(5)$ & $\mathrm{C} 34-\mathrm{C} 35-\mathrm{C} 40-\mathrm{O} 5$ & $4.4(14)$ \\
\hline $\mathrm{Au} 1-\mathrm{P} 1-\mathrm{C} 3-\mathrm{C} 8$ & $-26.2(8)$ & $\mathrm{C} 34-\mathrm{C} 35-\mathrm{C} 40-\mathrm{C} 39$ & $-178.6(9)$ \\
\hline $\mathrm{Au} 1-\mathrm{P} 1-\mathrm{C} 17-\mathrm{C} 18$ & $-170.1(6)$ & $\mathrm{C} 35-\mathrm{C} 36-\mathrm{C} 37-\mathrm{C} 38$ & $-2(2)$ \\
\hline $\mathrm{Au} 1-\mathrm{P} 1-\mathrm{C} 17-\mathrm{C} 22$ & $63.9(6)$ & $\mathrm{C} 36-\mathrm{C} 35-\mathrm{C} 40-\mathrm{O} 5$ & $179.2(10)$ \\
\hline $\mathrm{Au} 1-\mathrm{P} 1-\mathrm{C} 23-\mathrm{C} 24$ & $48.8(6)$ & $\mathrm{C} 36-\mathrm{C} 35-\mathrm{C} 40-\mathrm{C} 39$ & $-3.8(16)$ \\
\hline $\mathrm{Au} 1-\mathrm{P} 1-\mathrm{C} 23-\mathrm{C} 28$ & $-73.0(6)$ & $\mathrm{C} 36-\mathrm{C} 37-\mathrm{C} 38-\mathrm{C} 39$ & $-1(2)$ \\
\hline $\mathrm{Au} 2-\mathrm{P} 2-\mathrm{C} 29-\mathrm{C} 30$ & $-176.5(6)$ & $\mathrm{C} 37-\mathrm{C} 38-\mathrm{C} 39-\mathrm{C} 40$ & $1.1(18)$ \\
\hline $\mathrm{Au} 2-\mathrm{P} 2-\mathrm{C} 29-\mathrm{C} 34$ & $-0.7(10)$ & $\mathrm{C} 38-\mathrm{C} 39-\mathrm{C} 40-\mathrm{O} 5$ & $177.9(10)$ \\
\hline $\mathrm{Au} 2-\mathrm{P} 2-\mathrm{C} 43-\mathrm{C} 44$ & $-65.2(7)$ & $\mathrm{C} 38-\mathrm{C} 39-\mathrm{C} 40-\mathrm{C} 35$ & $1.2(16)$ \\
\hline $\mathrm{Au} 2-\mathrm{P} 2-\mathrm{C} 43-\mathrm{C} 48$ & $56.4(7)$ & $\mathrm{C} 40-\mathrm{C} 35-\mathrm{C} 36-\mathrm{O} 4$ & $-178.5(11)$ \\
\hline $\mathrm{Au} 2-\mathrm{P} 2-\mathrm{C} 49-\mathrm{C} 50$ & $-168.7(7)$ & $\mathrm{C} 40-\mathrm{C} 35-\mathrm{C} 36-\mathrm{C} 37$ & $4.4(18)$ \\
\hline $\mathrm{Au} 2-\mathrm{P} 2-\mathrm{C} 49-\mathrm{C} 54$ & $63.7(7)$ & $\mathrm{C} 41-\mathrm{O} 4-\mathrm{C} 36-\mathrm{C} 35$ & $122(2)$ \\
\hline $\mathrm{Au} 3-\mathrm{P} 3-\mathrm{C} 55-\mathrm{C} 56$ & $153.7(6)$ & $\mathrm{C} 41-\mathrm{O} 4-\mathrm{C} 36-\mathrm{C} 37$ & $-61(3)$ \\
\hline $\mathrm{Au} 3-\mathrm{P} 3-\mathrm{C} 55-\mathrm{C} 60$ & $-29.8(9)$ & $\mathrm{C} 42-\mathrm{O} 5-\mathrm{C} 40-\mathrm{C} 35$ & $175.4(11)$ \\
\hline $\mathrm{Au} 3-\mathrm{P} 3-\mathrm{C} 69-\mathrm{C} 70$ & $48.3(7)$ & $\mathrm{C} 42-\mathrm{O} 5-\mathrm{C} 40-\mathrm{C} 39$ & $-1.5(17)$ \\
\hline $\mathrm{Au} 3-\mathrm{P} 3-\mathrm{C} 69-\mathrm{C} 74$ & $-74.5(7)$ & $\mathrm{C} 43-\mathrm{P} 2-\mathrm{C} 29-\mathrm{C} 30$ & $55.1(8)$ \\
\hline $\mathrm{Au} 3-\mathrm{P} 3-\mathrm{C} 75-\mathrm{C} 76$ & $173.0(8)$ & $\mathrm{C} 43-\mathrm{P} 2-\mathrm{C} 29-\mathrm{C} 34$ & $-129.1(8)$ \\
\hline $\mathrm{Au} 3-\mathrm{P} 3-\mathrm{C} 75-\mathrm{C} 80$ & $39.9(13)$ & $\mathrm{C} 43-\mathrm{P} 2-\mathrm{C} 49-\mathrm{C} 50$ & $-48.9(8)$ \\
\hline $\mathrm{P} 1-\mathrm{C} 3-\mathrm{C} 4-\mathrm{C} 5$ & $172.4(7)$ & $\mathrm{C} 43-\mathrm{P} 2-\mathrm{C} 49-\mathrm{C} 54$ & $-176.6(7)$ \\
\hline $\mathrm{P} 1-\mathrm{C} 3-\mathrm{C} 8-\mathrm{C} 7$ & $-171.8(6)$ & $\mathrm{C} 43-\mathrm{C} 44-\mathrm{C} 45-\mathrm{C} 46$ & $-57.7(12)$ \\
\hline $\mathrm{P} 1-\mathrm{C} 3-\mathrm{C} 8-\mathrm{C} 9$ & $8.8(12)$ & $\mathrm{C} 44-\mathrm{C} 43-\mathrm{C} 48-\mathrm{C} 47$ & $-56.1(11)$ \\
\hline $\mathrm{P} 1-\mathrm{C} 17-\mathrm{C} 18-\mathrm{C} 19$ & $178.0(7)$ & $\mathrm{C} 44-\mathrm{C} 45-\mathrm{C} 46-\mathrm{C} 47$ & $55.9(12)$ \\
\hline $\mathrm{P} 1-\mathrm{C} 17-\mathrm{C} 22-\mathrm{C} 21$ & $-174.4(7)$ & $\mathrm{C} 45-\mathrm{C} 46-\mathrm{C} 47-\mathrm{C} 48$ & $-55.7(12)$ \\
\hline $\mathrm{P} 1-\mathrm{C} 23-\mathrm{C} 24-\mathrm{C} 25$ & $176.7(6)$ & $\mathrm{C} 46-\mathrm{C} 47-\mathrm{C} 48-\mathrm{C} 43$ & $56.2(12)$ \\
\hline $\mathrm{P} 1-\mathrm{C} 23-\mathrm{C} 28-\mathrm{C} 27$ & $-177.4(6)$ & $\mathrm{C} 48-\mathrm{C} 43-\mathrm{C} 44-\mathrm{C} 45$ & $57.5(10)$ \\
\hline $\mathrm{P} 2-\mathrm{C} 29-\mathrm{C} 30-\mathrm{C} 31$ & $172.5(8)$ & $\mathrm{C} 49-\mathrm{P} 2-\mathrm{C} 29-\mathrm{C} 30$ & $-59.2(8)$ \\
\hline $\mathrm{P} 2-\mathrm{C} 29-\mathrm{C} 34-\mathrm{C} 33$ & $-170.3(8)$ & $\mathrm{C} 49-\mathrm{P} 2-\mathrm{C} 29-\mathrm{C} 34$ & $116.6(9)$ \\
\hline $\mathrm{P} 2-\mathrm{C} 29-\mathrm{C} 34-\mathrm{C} 35$ & $12.0(14)$ & $\mathrm{C} 49-\mathrm{P} 2-\mathrm{C} 43-\mathrm{C} 44$ & $-179.7(6)$ \\
\hline $\mathrm{P} 2-\mathrm{C} 43-\mathrm{C} 44-\mathrm{C} 45$ & $180.0(7)$ & $\mathrm{C} 49-\mathrm{P} 2-\mathrm{C} 43-\mathrm{C} 48$ & $-58.1(8)$ \\
\hline $\mathrm{P} 2-\mathrm{C} 43-\mathrm{C} 48-\mathrm{C} 47$ & $-177.1(7)$ & $\mathrm{C} 49-\mathrm{C} 50-\mathrm{C} 51-\mathrm{C} 52$ & $57.7(12)$ \\
\hline $\mathrm{P} 2-\mathrm{C} 49-\mathrm{C} 50-\mathrm{C} 51$ & $174.5(7)$ & $\mathrm{C} 50-\mathrm{C} 49-\mathrm{C} 54-\mathrm{C} 53$ & $57.0(11)$ \\
\hline $\mathrm{P} 2-\mathrm{C} 49-\mathrm{C} 54-\mathrm{C} 53$ & $-171.1(7)$ & $\mathrm{C} 50-\mathrm{C} 51-\mathrm{C} 52-\mathrm{C} 53$ & $-55.9(13)$ \\
\hline P3-C55-C56-C57 & $177.7(7)$ & $\mathrm{C} 51-\mathrm{C} 52-\mathrm{C} 53-\mathrm{C} 54$ & $55.0(12)$ \\
\hline $\mathrm{P} 3-\mathrm{C} 55-\mathrm{C} 60-\mathrm{C} 59$ & $-175.4(7)$ & $\mathrm{C} 52-\mathrm{C} 53-\mathrm{C} 54-\mathrm{C} 49$ & $-55.5(11)$ \\
\hline $\mathrm{P} 3-\mathrm{C} 55-\mathrm{C} 60-\mathrm{C} 61$ & $9.0(12)$ & $\mathrm{C} 54-\mathrm{C} 49-\mathrm{C} 50-\mathrm{C} 51$ & $-58.2(11)$ \\
\hline $\mathrm{P} 3-\mathrm{C} 69-\mathrm{C} 70-\mathrm{C} 71$ & $176.8(7)$ & $\mathrm{C} 55-\mathrm{P} 3-\mathrm{C} 69-\mathrm{C} 70$ & $179.4(7)$ \\
\hline $\mathrm{P} 3-\mathrm{C} 69-\mathrm{C} 74-\mathrm{C} 73$ & $-176.1(7)$ & $\mathrm{C} 55-\mathrm{P} 3-\mathrm{C} 69-\mathrm{C} 74$ & $56.6(7)$ \\
\hline $\mathrm{P} 3-\mathrm{C} 75-\mathrm{C} 76-\mathrm{C} 77$ & $177.2(10)$ & $\mathrm{C} 55-\mathrm{P} 3-\mathrm{C} 75-\mathrm{C} 76$ & $40.3(10)$ \\
\hline
\end{tabular}




\begin{tabular}{|c|c|c|c|}
\hline $\mathrm{P} 3-\mathrm{C} 75-\mathrm{C} 80-\mathrm{C} 79$ & $-174.0(12)$ & $\mathrm{C} 55-\mathrm{P} 3-\mathrm{C} 75-\mathrm{C} 80$ & $-92.8(12)$ \\
\hline $\mathrm{O} 2-\mathrm{C} 10-\mathrm{C} 11-\mathrm{C} 12$ & $178.6(8)$ & $\mathrm{C} 55-\mathrm{C} 56-\mathrm{C} 57-\mathrm{C} 58$ & $-1.3(15)$ \\
\hline $\mathrm{O} 4-\mathrm{C} 36-\mathrm{C} 37-\mathrm{C} 38$ & $-179.3(12)$ & $\mathrm{C} 55-\mathrm{C} 60-\mathrm{C} 61-\mathrm{C} 62$ & $-105.9(11)$ \\
\hline $\mathrm{O} 6-\mathrm{C} 62-\mathrm{C} 63-\mathrm{C} 64$ & $180.0(9)$ & $\mathrm{C} 55-\mathrm{C} 60-\mathrm{C} 61-\mathrm{C} 66$ & $79.5(12)$ \\
\hline $\mathrm{C} 3-\mathrm{P} 1-\mathrm{C} 17-\mathrm{C} 18$ & $57.9(7)$ & $\mathrm{C} 56-\mathrm{C} 55-\mathrm{C} 60-\mathrm{C} 59$ & $1.1(13)$ \\
\hline $\mathrm{C} 3-\mathrm{P} 1-\mathrm{C} 17-\mathrm{C} 22$ & $-68.1(7)$ & $\mathrm{C} 56-\mathrm{C} 55-\mathrm{C} 60-\mathrm{C} 61$ & $-174.5(8)$ \\
\hline $\mathrm{C} 3-\mathrm{P} 1-\mathrm{C} 23-\mathrm{C} 24$ & $-178.5(6)$ & $\mathrm{C} 56-\mathrm{C} 57-\mathrm{C} 58-\mathrm{C} 59$ & $-0.4(15)$ \\
\hline $\mathrm{C} 3-\mathrm{P} 1-\mathrm{C} 23-\mathrm{C} 28$ & $59.7(7)$ & $\mathrm{C} 57-\mathrm{C} 58-\mathrm{C} 59-\mathrm{C} 60$ & $2.6(16)$ \\
\hline $\mathrm{C} 3-\mathrm{C} 4-\mathrm{C} 5-\mathrm{C} 6$ & $-0.8(14)$ & $\mathrm{C} 58-\mathrm{C} 59-\mathrm{C} 60-\mathrm{C} 55$ & $-2.9(15)$ \\
\hline $\mathrm{C} 3-\mathrm{C} 8-\mathrm{C} 9-\mathrm{C} 10$ & $75.4(11)$ & $\mathrm{C} 58-\mathrm{C} 59-\mathrm{C} 60-\mathrm{C} 61$ & $173.0(9)$ \\
\hline $\mathrm{C} 3-\mathrm{C} 8-\mathrm{C} 9-\mathrm{C} 14$ & $-110.5(10)$ & $\mathrm{C} 59-\mathrm{C} 60-\mathrm{C} 61-\mathrm{C} 62$ & $78.5(12)$ \\
\hline $\mathrm{C} 4-\mathrm{C} 3-\mathrm{C} 8-\mathrm{C} 7$ & $0.2(12)$ & $\mathrm{C} 59-\mathrm{C} 60-\mathrm{C} 61-\mathrm{C} 66$ & $-96.1(10)$ \\
\hline $\mathrm{C} 4-\mathrm{C} 3-\mathrm{C} 8-\mathrm{C} 9$ & $-179.2(8)$ & $\mathrm{C} 60-\mathrm{C} 55-\mathrm{C} 56-\mathrm{C} 57$ & $1.0(14)$ \\
\hline $\mathrm{C} 4-\mathrm{C} 5-\mathrm{C} 6-\mathrm{C} 7$ & $1.7(14)$ & $\mathrm{C} 60-\mathrm{C} 61-\mathrm{C} 62-\mathrm{O} 6$ & $4.7(13)$ \\
\hline $\mathrm{C} 5-\mathrm{C} 6-\mathrm{C} 7-\mathrm{C} 8$ & $-1.7(13)$ & $\mathrm{C} 60-\mathrm{C} 61-\mathrm{C} 62-\mathrm{C} 63$ & $-173.3(9)$ \\
\hline $\mathrm{C} 6-\mathrm{C} 7-\mathrm{C} 8-\mathrm{C} 3$ & $0.7(12)$ & $\mathrm{C} 60-\mathrm{C} 61-\mathrm{C} 66-\mathrm{O} 7$ & $-4.1(12)$ \\
\hline $\mathrm{C} 6-\mathrm{C} 7-\mathrm{C} 8-\mathrm{C} 9$ & $-179.8(8)$ & $\mathrm{C} 60-\mathrm{C} 61-\mathrm{C} 66-\mathrm{C} 65$ & $174.6(9)$ \\
\hline $\mathrm{C} 7-\mathrm{C} 8-\mathrm{C} 9-\mathrm{C} 10$ & $-104.1(9)$ & $\mathrm{C} 61-\mathrm{C} 62-\mathrm{C} 63-\mathrm{C} 64$ & $-2.2(15)$ \\
\hline $\mathrm{C} 7-\mathrm{C} 8-\mathrm{C} 9-\mathrm{C} 14$ & $70.1(10)$ & $\mathrm{C} 62-\mathrm{C} 61-\mathrm{C} 66-\mathrm{O} 7$ & $-178.9(8)$ \\
\hline $\mathrm{C} 8-\mathrm{C} 3-\mathrm{C} 4-\mathrm{C} 5$ & $-0.2(13)$ & $\mathrm{C} 62-\mathrm{C} 61-\mathrm{C} 66-\mathrm{C} 65$ & $-0.3(14)$ \\
\hline $\mathrm{C} 8-\mathrm{C} 9-\mathrm{C} 10-\mathrm{O} 2$ & $-3.3(11)$ & C62-C63-C64-C65 & $2.1(15)$ \\
\hline $\mathrm{C} 8-\mathrm{C} 9-\mathrm{C} 10-\mathrm{C} 11$ & $177.1(7)$ & C63-C64-C65-C66 & $-1.1(15)$ \\
\hline $\mathrm{C} 8-\mathrm{C} 9-\mathrm{C} 14-\mathrm{O} 3$ & $2.8(11)$ & $\mathrm{C} 64-\mathrm{C} 65-\mathrm{C} 66-\mathrm{O} 7$ & $178.6(9)$ \\
\hline $\mathrm{C} 8-\mathrm{C} 9-\mathrm{C} 14-\mathrm{C} 13$ & $-176.9(8)$ & $\mathrm{C} 64-\mathrm{C} 65-\mathrm{C} 66-\mathrm{C} 61$ & $0.1(14)$ \\
\hline $\mathrm{C} 9-\mathrm{C} 10-\mathrm{C} 11-\mathrm{C} 12$ & $-1.9(12)$ & $\mathrm{C} 66-\mathrm{C} 61-\mathrm{C} 62-\mathrm{O} 6$ & $179.3(8)$ \\
\hline $\mathrm{C} 10-\mathrm{C} 9-\mathrm{C} 14-\mathrm{O} 3$ & $177.1(7)$ & C66-C61-C62-C63 & $1.3(14)$ \\
\hline $\mathrm{C} 10-\mathrm{C} 9-\mathrm{C} 14-\mathrm{C} 13$ & $-2.5(12)$ & $\mathrm{C} 67-\mathrm{O} 6-\mathrm{C} 62-\mathrm{C} 61$ & $172.9(9)$ \\
\hline $\mathrm{C} 10-\mathrm{C} 11-\mathrm{C} 12-\mathrm{C} 13$ & $0.3(14)$ & $\mathrm{C} 67-\mathrm{O} 6-\mathrm{C} 62-\mathrm{C} 63$ & $-9.2(14)$ \\
\hline $\mathrm{C} 11-\mathrm{C} 12-\mathrm{C} 13-\mathrm{C} 14$ & $0.1(14)$ & $\mathrm{C} 68-\mathrm{O} 7-\mathrm{C} 66-\mathrm{C} 61$ & $-175.1(9)$ \\
\hline $\mathrm{C} 12-\mathrm{C} 13-\mathrm{C} 14-\mathrm{O} 3$ & $-178.5(8)$ & $\mathrm{C} 68-\mathrm{O} 7-\mathrm{C} 66-\mathrm{C} 65$ & $6.3(14)$ \\
\hline $\mathrm{C} 12-\mathrm{C} 13-\mathrm{C} 14-\mathrm{C} 9$ & $1.1(13)$ & $\mathrm{C} 69-\mathrm{P} 3-\mathrm{C} 55-\mathrm{C} 56$ & $29.3(8)$ \\
\hline $\mathrm{C} 14-\mathrm{C} 9-\mathrm{C} 10-\mathrm{O} 2$ & $-177.5(7)$ & $\mathrm{C} 69-\mathrm{P} 3-\mathrm{C} 55-\mathrm{C} 60$ & $-154.2(7)$ \\
\hline $\mathrm{C} 14-\mathrm{C} 9-\mathrm{C} 10-\mathrm{C} 11$ & $2.9(12)$ & $\mathrm{C} 69-\mathrm{P} 3-\mathrm{C} 75-\mathrm{C} 76$ & $-68.7(10)$ \\
\hline $\mathrm{C} 15-\mathrm{O} 2-\mathrm{C} 10-\mathrm{C} 9$ & $-172.4(7)$ & $\mathrm{C} 69-\mathrm{P} 3-\mathrm{C} 75-\mathrm{C} 80$ & $158.2(12)$ \\
\hline $\mathrm{C} 15-\mathrm{O} 2-\mathrm{C} 10-\mathrm{C} 11$ & $7.2(12)$ & $\mathrm{C} 69-\mathrm{C} 70-\mathrm{C} 71-\mathrm{C} 72$ & $55.0(12)$ \\
\hline $\mathrm{C} 16-\mathrm{O} 3-\mathrm{C} 14-\mathrm{C} 9$ & $170.5(8)$ & $\mathrm{C} 70-\mathrm{C} 69-\mathrm{C} 74-\mathrm{C} 73$ & $60.1(10)$ \\
\hline $\mathrm{C} 16-\mathrm{O} 3-\mathrm{C} 14-\mathrm{C} 13$ & $-9.8(12)$ & $\mathrm{C} 70-\mathrm{C} 71-\mathrm{C} 72-\mathrm{C} 73$ & $-51.6(12)$ \\
\hline $\mathrm{C} 17-\mathrm{P} 1-\mathrm{C} 3-\mathrm{C} 4$ & $-74.6(7)$ & $\mathrm{C} 71-\mathrm{C} 72-\mathrm{C} 73-\mathrm{C} 74$ & $51.4(12)$ \\
\hline $\mathrm{C} 17-\mathrm{P} 1-\mathrm{C} 3-\mathrm{C} 8$ & $97.4(7)$ & $\mathrm{C} 72-\mathrm{C} 73-\mathrm{C} 74-\mathrm{C} 69$ & $-55.8(12)$ \\
\hline $\mathrm{C} 17-\mathrm{P} 1-\mathrm{C} 23-\mathrm{C} 24$ & $-68.2(7)$ & $\mathrm{C} 74-\mathrm{C} 69-\mathrm{C} 70-\mathrm{C} 71$ & $-59.1(10)$ \\
\hline $\mathrm{C} 17-\mathrm{P} 1-\mathrm{C} 23-\mathrm{C} 28$ & $170.0(6)$ & $\mathrm{C} 75-\mathrm{P} 3-\mathrm{C} 55-\mathrm{C} 56$ & $-79.1(8)$ \\
\hline $\mathrm{C} 17-\mathrm{C} 18-\mathrm{C} 19-\mathrm{C} 20$ & $56.0(11)$ & $\mathrm{C} 75-\mathrm{P} 3-\mathrm{C} 55-\mathrm{C} 60$ & $97.4(8)$ \\
\hline $\mathrm{C} 18-\mathrm{C} 17-\mathrm{C} 22-\mathrm{C} 21$ & $56.1(10)$ & $\mathrm{C} 75-\mathrm{P} 3-\mathrm{C} 69-\mathrm{C} 70$ & $-71.0(8)$ \\
\hline $\mathrm{C} 18-\mathrm{C} 19-\mathrm{C} 20-\mathrm{C} 21$ & $-54.9(12)$ & $\mathrm{C} 75-\mathrm{P} 3-\mathrm{C} 69-\mathrm{C} 74$ & $166.2(7)$ \\
\hline $\mathrm{C} 19-\mathrm{C} 20-\mathrm{C} 21-\mathrm{C} 22$ & $53.1(12)$ & $\mathrm{C} 75-\mathrm{C} 76-\mathrm{C} 77-\mathrm{C} 78$ & $49(2)$ \\
\hline $\mathrm{C} 20-\mathrm{C} 21-\mathrm{C} 22-\mathrm{C} 17$ & $-54.4(12)$ & $\mathrm{C} 76-\mathrm{C} 75-\mathrm{C} 80-\mathrm{C} 79$ & $51(2)$ \\
\hline $\mathrm{C} 22-\mathrm{C} 17-\mathrm{C} 18-\mathrm{C} 19$ & $-56.2(10)$ & $\mathrm{C} 76-\mathrm{C} 77-\mathrm{C} 78-\mathrm{C} 79$ & $-51(2)$ \\
\hline $\mathrm{C} 23-\mathrm{P} 1-\mathrm{C} 3-\mathrm{C} 4$ & $36.4(8)$ & $\mathrm{C} 77-\mathrm{C} 78-\mathrm{C} 79-\mathrm{C} 80$ & $52(2)$ \\
\hline
\end{tabular}




$\mathrm{C} 23-\mathrm{P} 1-\mathrm{C} 3-\mathrm{C} 8$
$\mathrm{C} 23-\mathrm{P} 1-\mathrm{C} 17-\mathrm{C} 18$
$\mathrm{C} 23-\mathrm{P} 1-\mathrm{C} 17-\mathrm{C} 22$
$\mathrm{C} 23-\mathrm{C} 24-\mathrm{C} 25-\mathrm{C} 26$
$\mathrm{C} 24-\mathrm{C} 23-\mathrm{C} 28-\mathrm{C} 27$
$\mathrm{C} 24-\mathrm{C} 25-\mathrm{C} 26-\mathrm{C} 27$
$\mathrm{C} 25-\mathrm{C} 26-\mathrm{C} 27-\mathrm{C} 28$
$\mathrm{C} 26-\mathrm{C} 27-\mathrm{C} 28-\mathrm{C} 23$
$\mathrm{C} 28-\mathrm{C} 23-\mathrm{C} 24-\mathrm{C} 25$
$\mathrm{C} 29-\mathrm{P} 2-\mathrm{C} 43-\mathrm{C} 44$
$\mathrm{C} 29-\mathrm{P} 2-\mathrm{C} 43-\mathrm{C} 48$
$\mathrm{C} 29-\mathrm{P} 2-\mathrm{C} 49-\mathrm{C} 50$
$\mathrm{C} 29-\mathrm{P} 2-\mathrm{C} 49-\mathrm{C} 54$
$\mathrm{C} 29-\mathrm{C} 30-\mathrm{C} 31-\mathrm{C} 32$
$\mathrm{C} 29-\mathrm{C} 34-\mathrm{C} 35-\mathrm{C} 36$
$\mathrm{C} 29-\mathrm{C} 34-\mathrm{C} 35-\mathrm{C} 40$
$\mathrm{C} 30-\mathrm{C} 29-\mathrm{C} 34-\mathrm{C} 33$
$\mathrm{C} 30-\mathrm{C} 29-\mathrm{C} 34-\mathrm{C} 35$
C30-C $31-\mathrm{C} 32-\mathrm{C} 33$
C31-C $32-\mathrm{C} 33-\mathrm{C} 34$
C32-C $33-\mathrm{C} 34-\mathrm{C} 29$
C32-C $33-\mathrm{C} 34-\mathrm{C} 35$
C33-C $34-\mathrm{C} 35-\mathrm{C} 36$
C33-C $34-\mathrm{C} 35-\mathrm{C} 40$
C34-C $29-\mathrm{C} 30-\mathrm{C} 31$
C34-C $35-\mathrm{C} 36-\mathrm{O} 4$
C34-C $35-\mathrm{C} 36-\mathrm{C} 37$

$-151.5(7)$

$-52.0(7)$

$-178.0(6)$

$56.7(10)$

$59.8(9)$

$-53.5(10)$

$53.8(11)$

$-57.6(10)$

$-59.4(9)$

$68.8(7)$

$-169.6(7)$

$62.4(8)$

$-65.3(8)$

$-0.4(16)$

$85.6(13)$

$-99.8(12)$

$5.4(15)$

$-172.3(10)$

$2.5(17)$

$-0.5(18)$

$-3.5(17)$

$174.3(11)$

$-92.2(13)$

$82.5(13)$

$-3.5(15)$

$-3.6(16)$

$179.3(12)$
$-51(2)$

$-48.9(17)$

$10.1(11)$

$173.3(10)$

$51.0(10)$

$-65.6(10)$

$149.6(8)$

46.2 (11)

$-76.1(10)$

167.3 (9)

$17.2(10)$

$173.8(10)$

$-66.3(10)$

50.4 (10)

-126.4 (8)

-60.4 (11)

59.5 (9)

$176.2(8)$

$-65.1(11)$

$172.6(9)$

56.0 (10)

54.2 (11)

174.1 (8)

$-69.2(9)$

-103.8 (9)

$127.1(8)$ 